\title{
Singularity Conditions of 3T1R Parallel Manipulators with Identical Limb Structures
}

\author{
Semaan Amine ${ }^{\dagger}$, Mehdi Tale Masouleh ${ }^{\ddagger}$, Stéphane Caro*, Philippe Wenger ${ }^{\dagger}$, Clément Gosselin \\ $\dagger$ Institut de Recherche en Communications et Cybernétique de Nantes, UMR CNRS n 6597 \\ 1 rue de la Noë, 44321, Nantes, France \\ Email: \{semaan.amine, stephane.caro, philippe.wenger\}@irccyn.ec-nantes.fr \\ * Départment de génie mécanique, Université Laval \\ Québec, QC, Canada, G1V 0A6 \\ Email: mehdi.tale-masouleh.1@ulaval.ca, gosselin@gmc.ulaval.ca
}

\begin{abstract}
This paper deals with the singularity analysis of parallel manipulators with identical limb structures performing Schönflies motions, namely, three independent translations and one rotation about an axis of fixed direction (3TIR). Eleven architectures obtained from a recent type synthesis of such manipulators are analyzed. The constraint analysis shows that these architectures are all over-constrained and share some common properties between the actuation and the constraint wrenches. The singularities of such manipulators are examined through the singularity analysis of the 4- $\underline{R} U U$ parallel manipulator. A wrench graph representing the constraint wrenches and the actuation forces of the manipulator is introduced to formulate its superbracket. Grassmann-Cayley Algebra is used to obtain geometric singularity conditions. Based on the concept of wrench graph, Grassmann geometry is used to show the rank deficiency of the Jacobian matrix for the singularity conditions. Finally, this paper shows the general aspect of the obtained singularity conditions and their validity for $3 T 1 R$ parallel manipulators with identical limb structures.
\end{abstract}

\section{Introduction}

Lower-mobility Parallel Manipulators (PMs) are suitable for a wide range of applications that require fewer than six degrees of freedom (DOF). The singular configurations of lower-mobility PMs are critical poses characterized by either the loss of DOF, the gain of extra DOF or the loss of stiffness. The determination of singular configurations is thus a central issue in robotics due to their major effect on the robot performance. For a $(n<6)$-DOF PM, in a general configuration, the actuators apply a $n$-system of actuation wrenches whereas the legs apply a $(6-n)$-system of constraint wrenches, also known as platform constraints [1], on the moving platform.

\footnotetext{
*Address all correspondence to this author.
}

The Jacobian matrix $\mathbf{J}_{m}$ of a 6-DOF PM is a $6 \times 6$ matrix relating the linear and angular velocities of the end-effector to six input rates associated with six actuated joints. Zlatanov et al. [2] proposed an approach, using an input-output velocity relationship, to formulate a $6 \times 6 \mathbf{J}_{m}$ for 6 -DOF PMs and also for lower-mobility PMs whose limbs and endeffector have the same DOF. Nevertheless, their approach cannot provide a $6 \times 6 \mathbf{J}_{m}$ for a more general lower-mobility PM and thus it does not allow the examination of all singular configurations of such a PM. Based on the theory of reciprocal screws [3-7], Joshi and Tsai [8] developed a general methodology to perform the Jacobian analysis of lowermobility PMs, namely, to derive a $6 \times 6 \mathbf{J}_{m}$ providing information about both constraint and actuation wrench systems of such PMs. Accordingly, away from parallel singularities, the rows of $\mathbf{J}_{m}$ of a $(n<6)$-DOF PM are composed of $n$ linearly independent actuation wrenches plus $(6-n)$ linearly independent constraint wrenches.

The classification of singularities for lower-mobility PMs has stimulated the interest of many researchers $[2,8-$ 11]. In this paper, we adopt the classification proposed in [10], which is similar the one proposed in [8]. Accordingly, a lower-mobility PM can exhibit three different types of singularities: (i) limb singularities, (ii) platform singularities [10], also known as constraint singularities [1] and (iii) actuation singularities, also called architecture singularities [8]. A limb singularity is similar to the singularity of a serial manipulator. On the other hand, constraint and actuation singularities are referred to as parallel singularities and are related to the rank deficiency of $\mathbf{J}_{m}$. Linear algebra consists of a direct analysis of $\mathbf{J}_{m}$ by examining the vanishing conditions of its determinant. Mostly, the determinant of such a matrix is highly non linear and unwieldy to assess even with a computer algebra system. In that case, linear algebra fails to give satisfactory results, and therefore, the use of Grassmann-Cayley Algebra (GCA) [7, 12-15] or Grassmann Geometry (GG) [16-21] can be regarded as promising 
solutions.

The GCA is a systematic approach to obtain a bracket representation of the determinant of $\mathbf{J}_{m}$ called superbracket. By exploring this superbracket, all the parallel singularity conditions of a PM can be obtained either algebraically, geometrically or in a vector form. Ben-Horin et al. $[13,14]$ analyzed the singularities of 6-DOF PMs whose legs transmit six pure actuation forces, i.e., six finite lines, to the moving platform, by means of GCA. The singularity conditions are derived from a simplified expression of the superbracket, which is obtained by using the intersection points between actuation lines. Nevertheless, their method does not consider the intersection at infinity of two parallel finite lines. Moreover, it does not apply when a line at infinity is among the six Plücker lines of $\mathbf{J}_{m}$. Recently, Kanaan et al. [15] and Amine et al. [7, 22, 23] filled this gap by using some properties of projective geometry in order to formulate a superbracket with points and lines at infinity, and therefore, to extend the application of GCA to lower-mobility PMs.

In turn, GG is a geometric approach that provides a classification of the conditions under which a set of $n$ Plücker lines spans a variety of dimension lower than $n$. Merlet [16-18] proposed a framework based on GG, to analyze the singularities of PMs for which the rows of $\mathbf{J}_{m}$ are Plücker coordinate vectors of six finite lines. This method is based on a general classification of linear varieties. Tale Masouleh and Gosselin [20] followed the latter framework to analyze the singularities of 3T2R PMs, having a line at infinity among the rows of $\mathbf{J}_{m}$, using GG.

Schönflies Motion Generators (SMGs) [24] are manipulators performing three independent translations and one rotation about an axis of fixed direction, namely, 3T1R. This type of motion is required in a wide range of industrial pick and place operations such as the assembly of computer circuit boards. The GCA was used in $[7,15]$ to analyze the singularities of SMGs with an articulated moving platform and of SMGs with two limbs, two joints being actuated per limb.

This paper focuses on the singularity analysis of 3T1R PMs with Identical Limb Structures (ILS) using both GCA and GG as complementary approaches. The type synthesis of such PMs was performed in [6, 25, 26]. In [6], eleven architectures of four legged 3T1R PMs with ILS were obtained. Here, the singular configurations of these architectures are examined through the singularity analysis of the 4-RUU PM. Moreover, this paper aims to establish a conceptual approach to obtain the analogy between GCA and GG by providing a wrench graph, namely, a schematic representation, in the 3dimensional projective space, of the constraint and actuation wrenches of a given lower-mobility PM.

The remainder of this paper is organized as follows. First, some fundamental concepts of screw theory, projective space, GCA and GG are recalled. Then, eleven architectures of four legged 3T1R PMs with ILS are presented and their constraint analysis is performed. The singularity analysis is performed through an complete study of the 4RUU PM using GCA and GG and the concept of wrench graph. Accordingly, the three types of singularities-the constraint singularities, the actuation singularities and the limb singularities - of the 4-RUU PM are exhaustively examined and some singular configurations are illustrated. Finally, it is shown that the singularity conditions of the 4$\underline{\mathrm{R} U U} \mathrm{PM}$ are also true for the eleven architectures and some other 3T1R PMs.

\section{Preliminary Concepts \\ 2.1 Screw Theory}

Screw theory [3-6] is suitable for the type synthesis and the study of the instantaneous motion of PMs. A twist and a wrench are screws that represent the instantaneous motion of a rigid body and a system of forces and moments applied on a rigid body, respectively. A zero-pitch wrench, namely, a pure force, corresponds to the Plücker coordinate vector of a finite line in the 3 -dimensional projective space $\mathbb{P}_{3}$. In turn, an infinite-pitch wrench, namely, a pure moment, corresponds to the Plücker coordinate vector of a line at infinity in $\mathbb{P}_{3}$. It is noteworthy that finite lines and lines at infinity are projective lines, i.e., Plücker lines whose six components satisfy the Grassmann Plücker relation [27]. A $n$-screw system is a screw subspace whose basis is composed of $n$ screws.

\subsection{Projective Space}

The 3-dimensional projective space $\mathbb{P}_{3}$ can be interpreted as the affine space $\mathbb{R}^{3}$ in addition to the plane at infinity $\Omega_{\infty}$. It is noteworthy that the coordinates of a projective element are determined up to a scalar multiple. A projective point has four homogeneous coordinates whereas a projective line has six Plücker coordinates represented by its Plücker coordinate vector. The following properties highlight the relations between projective elements:

1. A finite point, $A$, is represented by its homogeneous coordinates vector $\mathrm{a}=\left(a_{1}, a_{2}, a_{3}, 1\right)^{T}$, the first three coordinates being its Cartesian coordinates in $\mathbb{R}^{3}$;

2. A finite line, $\mathcal{L}$, is represented by its Plücker coordinates vector ${ }^{1} F=(\mathbf{s} ; \mathbf{r} \times \mathbf{s})$; where $\mathbf{s}$ is the unit vector of $\mathcal{L}$, $\mathbf{r}$ is the position vector of any point on $\mathcal{L}$ and $(\mathbf{r} \times \mathbf{s})$ represents the moment of $\mathcal{L}$ with respect to the origin;

3. Let underlined points denote points at infinity. Any finite line, $F=(\mathbf{s} ; \mathbf{r} \times \mathbf{s})$, has a unique point at infinity $\underline{\mathbf{c}}=(\mathbf{s} ; 0)$. This point only depends on the line direction and is determined up to a scalar multiple. Accordingly, if $\mathrm{a}$ and $\mathrm{b}$ are two finite points on $F$, then $\underline{\mathrm{c}}=\mathrm{b}-\mathrm{a}$;

4. All finite lines directed along $\mathbf{S}$ intersect at one common point at infinity, namely, $\underline{\mathrm{c}}$;

5. All finite planes of normal vector $\mathbf{m}$, have a common line at infinity. This line is given by: $M=(\mathbf{0} ; \mathbf{m})$ and passes through the point at infinity of any finite line orthogonal to $\mathbf{m}$;

6. Two lines at infinity $M_{1}=\left(\mathbf{0} ; \mathbf{m}_{1}\right)$ and $M_{2}=\left(\mathbf{0} ; \mathbf{m}_{2}\right)$ intersect at a unique point at infinity $\underline{g}=\left(\mathbf{m}_{1} \times \mathbf{m}_{2} ; 0\right)$.

\footnotetext{
${ }^{1}$ In the scope of this paper, $(\mathbf{s} ; \mathbf{r} \times \mathbf{s})$ denotes a Plücker coordinate vector given by $\left[\begin{array}{c}\mathbf{S} \\ \mathbf{r} \times \mathbf{s}\end{array}\right]$.
} 


\subsection{Grassmann-Cayley Algebra and Superbracket}

The GCA was developed by H. Grassmann (1809-1877) as a calculus for linear varieties operating on extensors with the join " $\vee$ " and meet " $\wedge$ " operators. The latter are associated with the span and intersection of vector spaces of extensors characterized with their step. Indeed, points, lines and planes in the projective space are represented with extensors of step 1, 2 and 3, respectively. A Plücker line (extensor of step 2) can be obtained either from the join of two distinct projective points (extensors of step 1) or from the meet of two disctinct projective planes (extensors of step 3). GCA makes it possible to work at the symbolic level, and therefore, to produce coordinate-free algebraic expressions for the singularity conditions of spatial PMs. For further details on GCA, the reader is referred to $[13,14,27]$ and references therein.

The rows of $\mathbf{J}_{m}$ of a PM are usually Plücker coordinate vectors of six projective lines. The superjoin of these six vectors in $\mathbb{P}_{5}$ corresponds to the determinant of $\mathbf{J}_{m}$ up to a scalar multiple, which is the superbracket in GCA $\Lambda\left(V^{(2)}\right)$ [27]. Thus, a singularity occurs when this superbracket vanishes. The superbracket is an expression involving 12 points selected on the six lines and can be developed into a linear combination of 24 bracket monomials [13,28], each one being the product of three brackets of four projective points:

$$
[\mathrm{ab}, \mathrm{cd}, \mathrm{ef}, \mathrm{gh}, \mathrm{ij}, \mathrm{kl}]=\sum_{i=1}^{24} y_{i}
$$

where

$$
\begin{aligned}
& y_{1}=-[\mathrm{abcd}][\mathrm{efgi}][\mathrm{hjkl}] \quad y_{2}=[\mathrm{abcd}][\mathrm{efhi}][\mathrm{gjkl}] \\
& y_{3}=[\mathrm{abcd}][\mathrm{efgj}][\mathrm{hikl}] \quad y_{4}=-[\mathrm{abcd}][\mathrm{efhj}][\mathrm{gikl}] \\
& y_{5}=[\mathrm{abce}][\mathrm{dfgh}][\mathrm{ijkl}] \quad y_{6}=-[\text { abde }][\mathrm{cfgh}][\mathrm{ijkl}] \\
& y_{7}=-[\mathrm{abcf}][\mathrm{degh}][\mathrm{ijkl}] \quad y_{8}=[\mathrm{abdf}][\mathrm{cegh}][\mathrm{ijkl}] \\
& y_{9}=-[\mathrm{abce}][\mathrm{dghi}][\mathrm{fjkl}] \quad y_{10}=[\mathrm{abde}][\mathrm{cghi}][\mathrm{fjkl}] \\
& y_{11}=[\mathrm{abcf}][\mathrm{dghi}][\mathrm{ejkl}] \quad y_{12}=[\mathrm{abce}][\mathrm{dghj}][\mathrm{fikl}] \\
& y_{13}=-[\operatorname{abdf}][\mathrm{cghi}][\mathrm{ejkl}] \quad y_{14}=-[\mathrm{abde}][\mathrm{cghj}][\mathrm{fikl}] \\
& y_{15}=-[\text { abcf }][\operatorname{dghj}]\left[\text { eikl] } \quad y_{16}=[\mathrm{abdf}][\mathrm{cghj}][\mathrm{eikl}]\right. \\
& y_{17}=[\mathrm{abcg}][\operatorname{def} \mathrm{i}][\mathrm{hjkl}] \quad y_{18}=-[\mathrm{abdg}][\operatorname{cef} \mathrm{i}][\mathrm{hj} \mathrm{kl}] \\
& y_{19}=-[\mathrm{abch}][\operatorname{defi}][\mathrm{gjkl}] \quad y_{20}=-[\mathrm{abcg}][\operatorname{defj}][\mathrm{hikl}] \\
& y_{21}=[\mathrm{abdh}][\operatorname{cefi}][\mathrm{gjkl}] \quad y_{22}=[\mathrm{abdg}][\operatorname{cefj}][\mathrm{hikl}] \\
& y_{23}=[\text { abch }][\text { def } j][\text { gikl }] \quad y_{24}=-[\text { abdh }][\text { cef } j][\text { gikl }]
\end{aligned}
$$

\begin{tabular}{|c|c|c|c|}
\hline Rank & Class & & Linear line variety \\
\hline 0 & empty set & & $\emptyset$ \\
\hline 1 & point & & a line in the $3-\mathrm{D}$ space \\
\hline \multirow[t]{2}{*}{2} & \multirow[t]{2}{*}{ lines } & $(2 a)$ & a pair of skew lines \\
\hline & & $(2 b)$ & a planar pencil of lines \\
\hline \multirow[t]{4}{*}{3} & \multirow[t]{4}{*}{ planes } & (3a) & a regulus \\
\hline & & $(3 b)$ & $\begin{array}{l}\text { the union of two planar pencils } \\
\text { having a line in common } \\
\text { but lying in distinct planes } \\
\text { and with distinct centers }\end{array}$ \\
\hline & & $(3 c)$ & all lines through a point \\
\hline & & $(3 \mathrm{~d})$ & all lines in a plane \\
\hline \multirow[t]{4}{*}{4} & \multirow[t]{4}{*}{ congruences } & $(4 a)$ & elliptic congruence \\
\hline & & $(4 b)$ & hyperbolic congruence \\
\hline & & $(4 c)$ & parabolic congruence \\
\hline & & $(4 d)$ & degenerate congruence \\
\hline \multirow[t]{2}{*}{5} & \multirow[t]{2}{*}{ complexes } & (5a) & $\begin{array}{l}\text { non-singular complex; } \\
\text { generated by five skew lines }\end{array}$ \\
\hline & & $(5 b)$ & $\begin{array}{l}\text { singular complex; all the } \\
\text { lines meeting one given line }\end{array}$ \\
\hline
\end{tabular}

A bracket $[a b c d]$ is null if and only if (iff) the projective points $a, b, c$ and $d$ are coplanar. The bracket of four projective points is defined as the determinant of the matrix whose columns are the homogeneous coordinates of these points. It is noteworthy that a bracket containing one finite point and three distinct points at infinity does not depend on the finite point. Indeed, let $g, \underline{i}, j$ and $\underline{\mathrm{k}}$ be one given finite point and three disctinct points at infinity, respectively. Then,

$$
[\mathrm{g} \underline{\underline{\mathrm{i}}} \underline{\mathrm{k}} \underline{\mathrm{j}}]=\left|\begin{array}{cccc}
g_{1} & i_{1} & k_{1} & j_{1} \\
g_{2} & i_{2} & k_{2} & j_{2} \\
g_{3} & i_{3} & k_{3} & j_{3} \\
1 & 0 & 0 & 0
\end{array}\right|=\left|\begin{array}{lll}
i_{1} & k_{1} & j_{1} \\
i_{2} & k_{2} & j_{2} \\
i_{3} & k_{3} & j_{3}
\end{array}\right|=[\underline{\underline{i}} \underline{\mathrm{k}} \underline{\mathrm{j}}]=[\mathrm{x} \underline{\underline{i}} \underline{\mathrm{k}} \underline{\mathrm{j}}]
$$

where $\mathrm{x}$ can be any finite point.
Table 1. Grassmann varieties [16-18].

\subsection{Grassmann Geometry}

A general classification of linear line varieties can be found in [16-18] and is summarized in Table 1. Since a projective line can be either a finite line or a line at infinity, the foregoing classification does apply for a linear line variety in which some lines at infinity may appear. In what follows, some particularities with points and lines at infinity are highlighted based on Table 1 .

\subsubsection{Planar Pencil of Lines, condition (2b)}

A planar pencil of lines spans a linear line variety of dimension 2, including all coplanar lines through a common point. When this point is at infinity, two sub-cases could be noticed:

(2b1) All the lines at infinity (in the plane $\Omega_{\infty}$ ) passing through a point at infinity;

(2b2) All the lines of a finite plane passing through a point at infinity. Let us consider a finite plane $\Omega$ of normal vector $(\mathbf{p} \times \mathbf{s})$. All finite lines $F_{i}=\left(\mathbf{s} ; \mathbf{r}_{i} \times \mathbf{s}\right)$ in $\Omega$ directed along $\mathbf{s}$, as well as the unique line at infinity of $\Omega, M=$ $(\mathbf{0} ; \mathbf{p} \times \mathbf{s})$, pass through point $\underline{\mathrm{t}}=(\mathbf{s} ; 0)$. Thus, the planar pencil, through point $\underline{\mathrm{t}}$, including $M$ in addition to the set of all the previous $F_{i}$, corresponds to a linear line variety of dimension 2 whose basis can be computed of any 


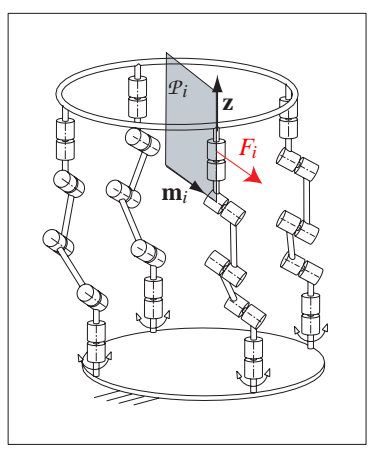

(a) $4-\underline{R} \underline{R} \tilde{R} \tilde{R} \tilde{R} \dot{R}$

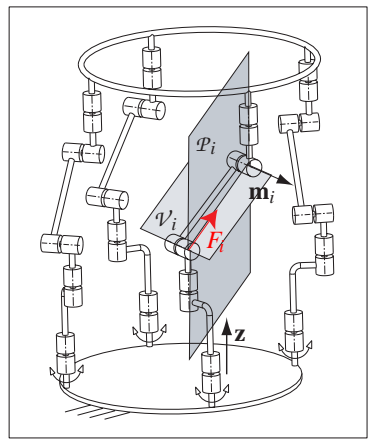

(e) $4-\underline{R} \underline{R} \hat{R} \bar{R} \bar{R} \dot{R}$

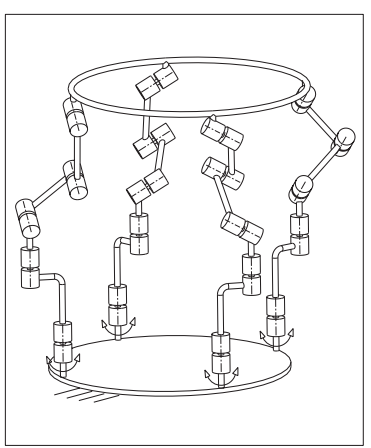

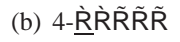

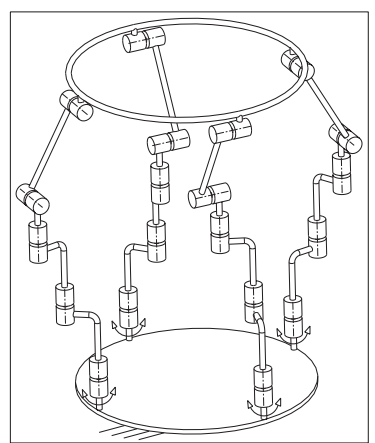

(f) $4-\underline{R} \dot{R} \hat{R} \bar{R} \bar{R}$

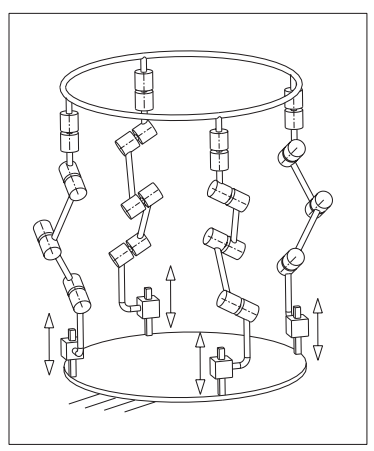

(c) $4-\underline{P} \tilde{R} \tilde{R} \tilde{R} \dot{R}$

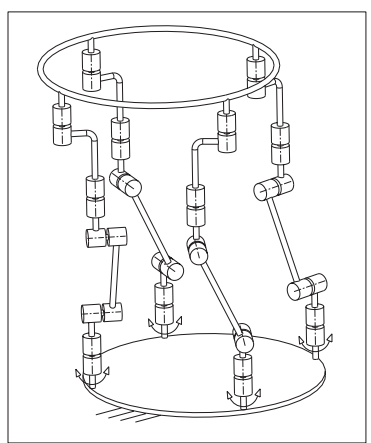

(g) $4-\underline{R} \bar{R} \bar{R} \dot{R} \dot{R}$

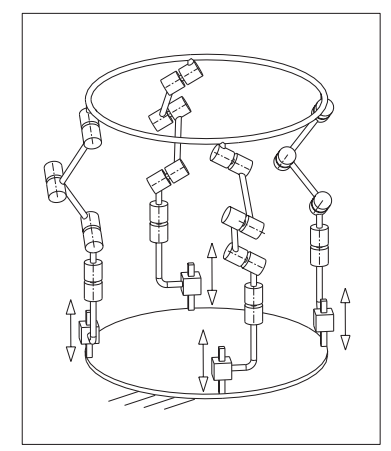

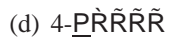

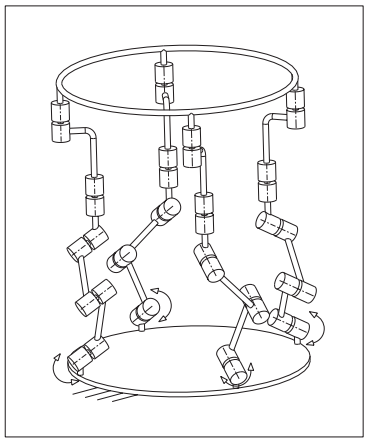

(h) 4- $\underline{\tilde{R}} \tilde{R} \tilde{R} \dot{R} \dot{R}$

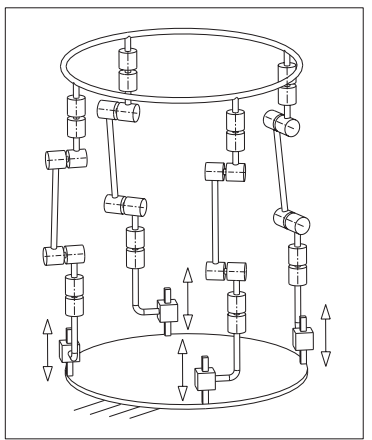

(i) $4-\underline{P} \hat{R} \bar{R} \bar{R} \dot{R}$

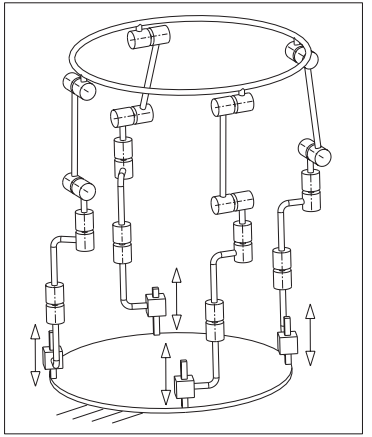

(j) 4-P̈R̀R̀ $\bar{R} \bar{R}$

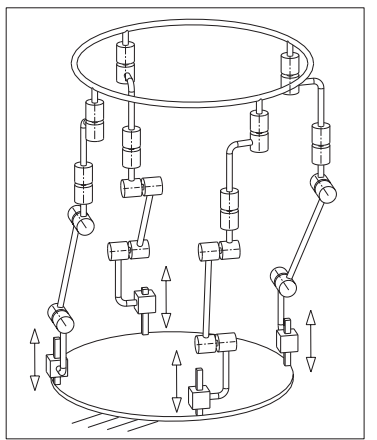

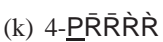

Fig. 1. 3T1R PMs with identical limb structures [6].

two among its distinct lines. Hence, if $F_{i}=\left(\mathbf{s} ; \mathbf{r}_{i} \times \mathbf{s}\right)$ and $F_{j}=\left(\mathbf{s} ; \mathbf{r}_{j} \times \mathbf{s}\right)$, then $\operatorname{span}\left(F_{i}, F_{j}\right)=\operatorname{span}\left(F_{i}, M_{i j}\right)$ where $M_{i j}=\left(\mathbf{0} ; \mathbf{s} \times \mathbf{r}_{i j}\right)$ and $\mathbf{r}_{i j}$ is the unit vector of a finite line non-parallel to $\mathbf{s}$ and crossing both $F_{i}$ and $F_{j}$.

\subsubsection{All Lines in a Plane, condition (3d)}

All lines in a plane span a variety of dimension 3.

(3d1) All lines at infinity lie in the plane at infinity $\Omega_{\infty}$. Thus, they generate a linear line variety of dimension 3 .

\section{Constraint Analysis of 3T1R PMs with ILS}

The type synthesis of 3T1R PMs performed in [6] has led to eleven architectures of 3T1R PMs with identical limb structures shown in Fig. 1. The foregoing PMs are supposed to produce three translational DOF and one rotational DOF about an axis directed along $\mathbf{z}$. For the architectures of Fig. 1:

1. $\dot{R}$ is a revolute joint of axis parallel to $\mathbf{z}$;
2. $\overline{\mathrm{R}}$ is a revolute joint of axis parallel to the horizontal plane of the fixed base;

3. $\tilde{R}$ is a revolute joint whose axis is neither parallel to $\mathbf{z}$ nor to the horizontal plane of the fixed base;

4. All $\bar{R}$-joints (all $\tilde{R}$-joints, respectively) in a given limb have parallel axes;

5. In the $i$ th limb, let $\mathbf{m}_{i}$ denote the direction vector of the revolute joint axes that are not parallel to $\mathbf{z}$

6. The first joint of each limb is actuated.

\subsection{Constraint Wrench System of 3T1R PMs with ILS}

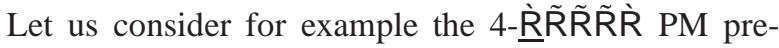
sented in Fig. 1(a). Each limb $l_{i}$ of this PM applies one constraint moment $M_{i}=\left(\mathbf{0} ; \mathbf{m}_{i} \times \mathbf{z}\right)$ on the moving platform. This moment is reciprocal to the twists associated with the limb joints. The four constraint moments $M_{i}$, applied by the four limbs, span the constraint wrench system of the PM, 
namely,

$$
\mathrm{W}^{c}=\operatorname{span}\left(M_{i}\right) \quad ; \quad M_{i}=\left(\mathbf{0} ; \mathbf{m}_{i} \times \mathbf{z}\right) \quad ; \quad i=1, \ldots, 4
$$

Since vectors $\mathbf{m}_{i} \times \mathbf{z}$ have a common orthogonal vector, i.e., $\mathbf{z}, \mathrm{W}^{c}$ is a 2-system in a non-singular configuration. The legs of the 4- $\underline{R} \tilde{R} \tilde{R} \tilde{R} \dot{R}$ PM can each apply a constraint moment but altogether they constrain only two DOF, namely, two rotations of the moving platform. Thus, this PM is an overconstrained SMG.

The expression of the constraint wrench system given in Eqn. (3) applies for any given architecture of Fig. 1.

\subsection{Actuation Wrench System of 3T1R PMs with ILS}

Let us consider that the actuated joint of a limb of a given PM of Fig. 1 is locked. Let $\mathrm{V}^{c}$ denote the wrench system reciprocal to the unactuated joint of the limb. Generally, $\operatorname{dim}\left(\mathrm{V}^{c}\right)=\operatorname{dim}\left(\mathrm{W}^{c}\right)+1$ and $\mathrm{V}^{c}$ contains $\mathrm{W}^{c}$ plus a set of some additional wrenches. Then, the actuation wrench can be selected as one of these additional wrenches. Based on the locus of the limb actuation wrench, the architectures of Fig. 1 can be classified into two types.

\subsubsection{Type 1: Figs. 1(a)-1(d)}

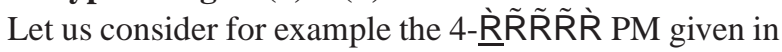
Fig. 1(a). By locking the actuated joint of a given limb $l_{i}$, a set of additional constraint wrenches appears. These additional wrenches are zero-pitch wrenches whose axes are parallel to $\mathbf{m}_{i}$ and cross the axis of the unactuated $\dot{\mathrm{R}}_{\text {-joint. Thus, }}$ the limb actuation force can be selected as $F_{i}=\left(\mathbf{m}_{i} ; \mathbf{r}_{C_{i}} \times \mathbf{m}_{i}\right)$ where $C_{i}$ can be any point on the axis of the unactuated $\dot{\mathrm{R}}$ joint of the limb. Consequently, the locus of $F_{i}$ is a plane $\mathcal{P}_{i}$ containing the axis of the unactuated $\dot{R}$-joint of the limb plus the unit vector $\mathbf{m}_{i}$.

The actuation force for any limb of the PMs of Figs. 1(b)-1(d) can be determined similarly to the 4-

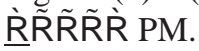

\subsubsection{Type 2: Figs. 1(e)-1(k)}

Let us consider for example the 4- $\underline{\mathrm{R}} \mathrm{R} \overline{\mathrm{R}} \overline{\mathrm{R}} \mathrm{R}$ PM given in Fig. 1(e). Let $\mathcal{P}_{i}\left(\mathcal{V}_{i}\right.$, respectively) denote the plane defined by the axes of the two unactuated $\mathrm{R}$-joints (the two $\overline{\mathrm{R}}$-joints, respectively) of the $i$ th limb. The limb actuation force is expressed as $F_{i}=\left(\mathbf{f}_{i} ; \mathbf{r}_{C_{i}} \times \mathbf{f}_{i}\right)$ where $\mathbf{f}_{i}$ is the unit vector of $\mathcal{P}_{i} \cap \mathcal{V}_{i}$, namely, the intersection line of planes $\mathcal{P}_{i}$ and $\mathcal{V}_{i}$ and $C_{i}$ can be any point on this line.

The actuation force for any limb of the PMs of Figs. 1(f)-1(k) can be determined similarly to the 4- $\underline{R} \dot{R} \bar{R} \bar{R} \dot{R}$ PM.

In a non-singular configuration, the actuation wrench system of a 3T1R PM with ILS is a 4-system expressed as:

$$
\mathrm{W}^{a}=\operatorname{span}\left(F_{i}\right) \quad ; \quad i=1, \ldots, 4
$$

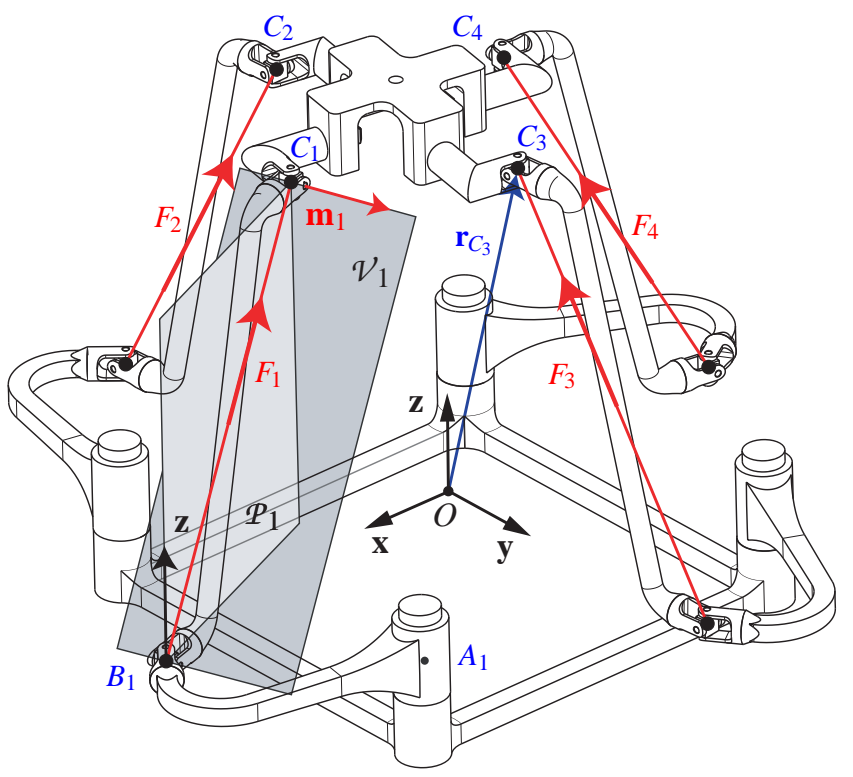

Fig. 2. The 4-RUU PM.

\subsubsection{Relation between Two Actuation Forces}

Let us consider two actuation forces $F_{i}$ and $F_{j}$ provided by two limbs of a given 3T1R PM with ILS. It can be noticed that, for the previous two types of 3T1R PMs with ILS, the actuation force $F_{i}$ of any limb lies in a plane $\mathcal{P}_{i}$ containing the unit vector $\mathbf{z}$. Generally, such two planes $\mathcal{P}_{i}$ and $\mathcal{P}_{j}(i \neq j)$ intersect at a finite line parallel to $\mathbf{z}$, namely, $\mathcal{T}_{i j}=\left(\mathcal{P}_{i} \cap \mathcal{P}_{j}\right)$. Therefore, for $i \neq j$, one can find a finite line $\mathcal{T}_{i j}$ directed along $\mathbf{Z}$ and crossing the two actuation forces $F_{i}$ and $F_{j}$.

\section{Jacobian Matrix of the 4-RUU PM}

\subsection{Architecture Review and Kinematic Modeling}

Figure 2 represents a CAD model of a 4-ㅡUU PM similar to the architecture of Fig. 1(e), which consists of a fixed base and a moving platform connected to each other with four identical RUU limbs. The input of the mechanism is provided by four revolute actuators attached to the base. The geometric characteristics of each limb are:

1. Each limb is composed of five revolute joints such that the second and the third ones, as well as the fourth and the fifth ones, are built with intersecting and perpendicular axes and are thus assimilated to $U$-joints of central points $B_{i}$ and $C_{i}$, respectively;

2. The axes of the third and the fourth revolute joints of the $i$ th limb are directed along $\mathbf{m}_{i}$. These axes form a plane $\mathcal{V}_{i}$. Let $\mathbf{f}_{i}$ be the unit vector directed along line $B_{i} C_{i}$. Thus, plane $\mathcal{V}_{i}$ has $\left(\mathbf{m}_{i} \times \mathbf{f}_{i}\right)$ as normal vector;

3. The axes of the first, second and fifth revolute joints of the $i$ th limb are directed along $\mathbf{z}$. Plane $\mathcal{P}_{i}$, defined by the axes of the second and the last revolute joints, has $\left(\mathbf{z} \times \mathbf{f}_{i}\right)=\mathbf{m}_{i}$ as normal vector. It should be noted that plane $\mathcal{P}_{i}$ degenerates into a line if $\mathbf{f}_{i}$ is parallel to $\mathbf{z}$, which is a limb singularity. 
Under these geometric constraints, the moving platform of the 4-RUU PM has a 3T1R motion pattern. However, in some configurations, these geometric constraints may be lost and the manipulator may switch to another motion mode (see Sec. 6.2 and Fig. 6). In such a mode, the axis of the last Rjoint in each limb is no longer parallel to $\mathbf{z}$.

\subsection{Constraint Analysis}

The constraint wrench system $\mathrm{W}_{4 R U U}^{c}$ of the 4-RUU PM can be obtained from Eqn. (3), namely,

$$
\mathrm{W}_{4 R U U}^{c}=\operatorname{span}\left(M_{i}\right) ; i=1, \ldots, 4
$$

Accordingly, it is an over-constrained SMG. The actuation force of the $i$ th limb is expressed as $F_{i}=\left(\mathbf{f}_{i} ; \mathbf{r}_{C_{i}} \times \mathbf{f}_{i}\right)$ where $\mathbf{f}_{i}$ is the unit vector of $\left(B_{i} C_{i}=\mathcal{P}_{i} \cap \mathcal{V}_{i}\right)$ and $\mathbf{r}_{C_{i}}$ is the position vector of point $C_{i}$. In a non-singular configuration, the actuation wrench system of the PM is a 4-system expressed as:

$$
\mathrm{W}_{4 R U U}^{a}=\operatorname{span}\left(F_{i}\right) ; i=1, \ldots, 4
$$

The rows of $\mathbf{J}_{m}$ of the 4-RUU PM are composed of four independent zero-pitch wrenches within $\mathrm{W}_{4 R U U}^{a}$ plus two independent infinite-pitch wrenches within $\mathrm{W}_{4 R U U}^{c}$. However, a parallel singularity occurs when the system spanned by the four actuation forces and the four constraint moments becomes a $(n<6)$-system.

\section{Limb Singularities of the 4-RUU PM}

A limb singularity is similar to the singularity of a serial manipulator. It occurs for the 4-RUU PM when a limb kinematic screw system (twist system) degenerates. Consequently, the platform loses one DOF in such a configuration. Let us consider the twist system of the $i$ th limb. It can be expressed in matrix form as follows:

$$
\left[\begin{array}{ccccc}
\mathbf{z} & \mathbf{z} & \mathbf{m}_{i} & \mathbf{m}_{i} & \mathbf{z} \\
\mathbf{z} \times \mathbf{r}_{A_{i}} & \mathbf{z} \times \mathbf{r}_{B_{i}} & \mathbf{m}_{i} \times \mathbf{r}_{B_{i}} & \mathbf{m}_{i} \times \mathbf{r}_{C_{i}} & \mathbf{z} \times \mathbf{r}_{C_{i}}
\end{array}\right]
$$

After some linear transformations, Eqn. (7) becomes:

$$
\left[\begin{array}{ccccc}
\mathbf{z} & \mathbf{m}_{i} & \mathbf{0} & \mathbf{0} & \mathbf{0} \\
\mathbf{z} \times \mathbf{r}_{A_{i}} & \mathbf{m}_{i} \times \mathbf{r}_{B_{i}} & \mathbf{z} \times\left(\mathbf{r}_{B_{i}}-\mathbf{r}_{A_{i}}\right) & \mathbf{z} \times\left(\mathbf{r}_{C_{i}}-\mathbf{r}_{A_{i}}\right) & \mathbf{m}_{i} \times \mathbf{f}_{i}
\end{array}\right]
$$

where $\mathbf{f}_{i}=\mathbf{r}_{C_{i}}-\mathbf{r}_{B_{i}}$. Since $\mathbf{z}$ and $\mathbf{m}_{i}$ are two independent vectors, this matrix is rank deficient whenever its last three columns (corresponding to infinite-pitch twists) become linearly dependent. On the other hand, in any robot configuration one has: $A_{i}, B_{i}$ and $C_{i}$ are three distinct points, $A_{i} B_{i} \perp \mathbf{z}$ and $\mathbf{m}_{i} \nVdash \mathbf{f}_{i}$. Thus, the $i$ th limb of the 4-RUU PM may exhibit a limb singularity whenever:

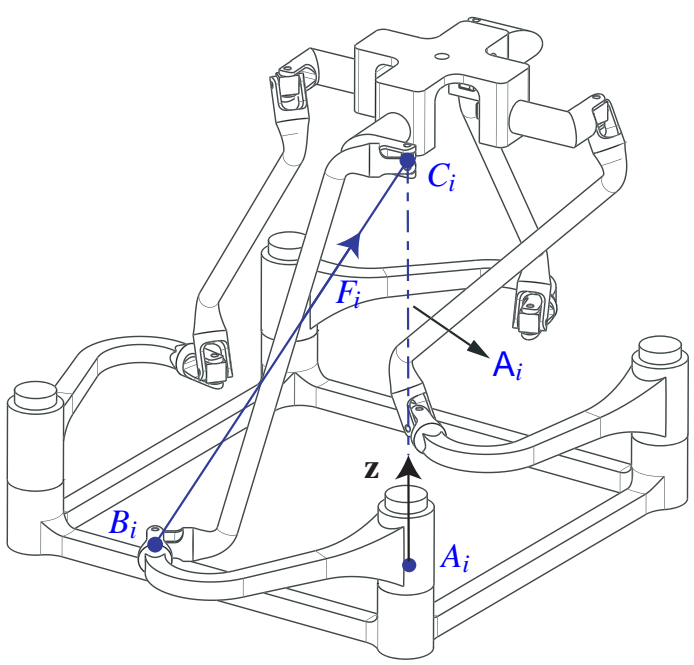

Fig. 3. A limb singularity, condition a.1.

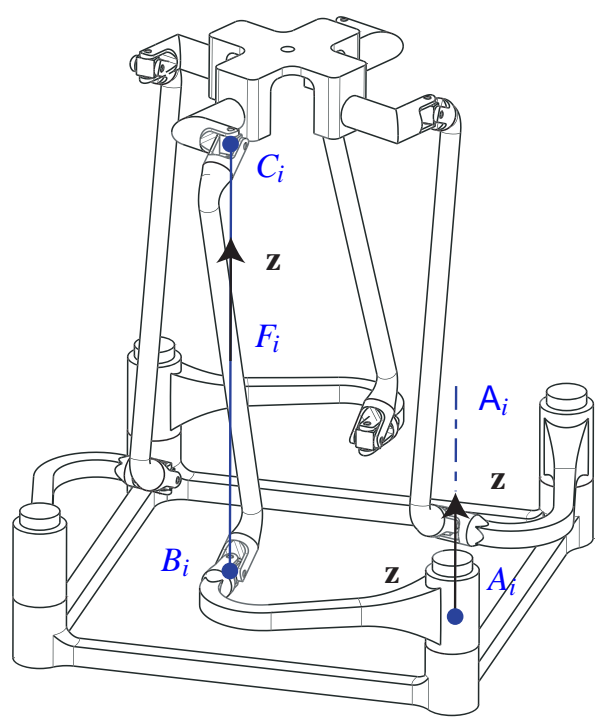

Fig. 4. A limb singularity, condition a.2.

a.1 $\left(\mathbf{r}_{C_{i}}-\mathbf{r}_{A_{i}}\right)\left\|\mathbf{z} \Leftrightarrow A_{i} C_{i}\right\| \mathbf{z}$. In that case, $F_{i}=\left(\mathbf{f}_{i} ; \mathbf{r}_{C_{i}} \times \mathbf{f}_{i}\right)$ crosses $\mathrm{A}_{i}$ at point $C_{i}$. As a result, $F_{i}$ acts as a constraint force and the 4-RUU PM loses the translational DOF along $\mathbf{f}_{i}$. Figure 3 illustrates such a configuration;

a.2 $\left(\mathbf{r}_{C_{i}}-\mathbf{r}_{B_{i}}\right)\left\|\mathbf{z} \Leftrightarrow \mathbf{f}_{i}\right\| \mathbf{z}$ as shown in Fig. 4. In that case, $F_{i}$ crosses $\mathrm{A}_{i}$ at infinity, i.e., at point $\underline{\mathrm{j}}=(\mathbf{z} ; 0)$. Consequently, the 4-RUU PM loses the translational DOF along $\mathbf{z}$.

If several limb singularities occur simultaneously, then the robot may lose several DOF. Besides, if some limb singularities and a constraint singularity occur simultaneously, then the lower-mobility PM may lose some allowed motions and gain some other limited motions at the same time. As a consequence, this may lead to unwanted changes on the motion pattern of the PM. 
6 Singularity Analysis of the 4-RUU PM using Grassmann-Cayley Algebra

In order to formulate a superbracket expression of the 4-iUU PM, one could represent its different wrenches in $\mathbb{P}_{3}$ and then select two points on each Plücker line of $\mathbf{J}_{m}$. A finite line (pure force) can be represented in the superbracket either by two finite points or by one finite point and its unique point at infinity. In turn, a line at infinity can be represented by two points at infinity. However, the selection of the foregoing points must highlight as much as possible geometric relations (coincidence, parallelism, orthogonality, intersection and so on) between the wrenches in order to obtain a simplified expression of the superbracket.

As shown in Fig. 2, each actuation force of the 4RUU PM can be expressed as $F_{i}=\left(\mathcal{P}_{i} \cap \mathcal{V}_{i}\right), i=1, \ldots, 4$, plane $\mathcal{P}_{i}$ being of normal $\left(\mathbf{z} \times \mathbf{f}_{i}\right)=\mathbf{m}_{i}$ while plane $\mathcal{V}_{i}$ is of normal $\mathbf{m}_{i} \times \mathbf{f}_{i}$. On the other hand, in a general configuration, two planes $\mathcal{P}_{i}$ and $\mathcal{P}_{j}(i \neq j)$ intersect at a finite line, namely, $\mathcal{T}_{i j}=\left(\mathcal{P}_{i} \cap \mathcal{P}_{j}\right)$. Such a line is orthogonal to both vectors $\mathbf{z} \times \mathbf{f}_{i}$ and $\mathbf{z} \times \mathbf{f}_{j}$ and is thus directed along $\mathbf{z}$. Therefore, for $i \neq j$, one can find a line $\mathcal{T}_{i j}=\left(\mathscr{P}_{i} \cap \mathcal{P}_{j}\right)$ directed along $\mathbf{z}$ and crossing the two actuation forces $F_{i}$ and $F_{j}$. In this vein, let a and $\mathrm{c}$ be the intersection points of $\mathcal{T}_{12}$ with $F_{1}$ and $F_{2}$, respectively. Likewise, let e and $\mathrm{g}$ be the intersection points of $\mathcal{T}_{34}$ with $F_{3}$ and $F_{4}$, respectively.

On the other hand, let $\underline{\mathrm{b}}=\left(\mathbf{f}_{1} ; 0\right), \underline{\mathrm{d}}=\left(\mathbf{f}_{2} ; 0\right), \underline{\mathbf{f}}=\left(\mathbf{f}_{3} ; 0\right)$ and $\underline{\underline{h}}=\left(\mathbf{f}_{4} ; 0\right)$. Accordingly, the four actuation forces can be expressed as:

$$
F_{1}=\mathrm{ab} \quad ; \quad F_{2}=\mathrm{c} \underline{\mathrm{d}} \quad ; \quad F_{3}=\mathrm{e} \underline{\mathrm{f}} \quad ; \quad F_{4}=\mathrm{g} \underline{\mathrm{h}}
$$

Now let $\underline{x}=(\mathbf{x} ; 0)$ and $\underline{y}=(\mathbf{y} ; 0)$. Hence, line $\underline{x} \underline{y}$ collects all points at infinity corresponding to directions orthogonal to $\mathbf{z}$. Let $\underline{j}=(\mathbf{z} ; 0), \underline{\underline{i}}=\left(\mathbf{m}_{1} ; 0\right), \underline{\mathrm{k}}=\left(\mathbf{m}_{2} ; 0\right)$, $\underline{\underline{l}}=\left(\mathbf{m}_{3} ; 0\right)$ and $\underline{\underline{m}}=\left(\mathbf{m}_{4} ; 0\right)$. Accordingly, the four constraint moments are expressed as:

$$
M_{1}=\underline{\mathrm{i}} \underline{\mathrm{j}} \quad ; \quad M_{2}=\underline{\mathrm{k}} \underline{\mathrm{j}} \quad ; \quad M_{3}=\underline{\mathrm{l}} \underline{\mathrm{j}} ; M_{4}=\underline{\mathrm{m}} \underline{\mathrm{j}}
$$

where $\underline{\underline{i}}, \underline{\mathrm{k}}, \underline{\mathrm{I}}$ and $\underline{\mathrm{m}}$ belong to $\underline{\mathrm{x}} \mathrm{y}$. A wrench graph, representing the projective lines associated with the wrenches of the 4-․ㅡU PM in $\mathbb{P}_{3}$, is given in Fig. 5.

\subsection{Superbracket Decomposition}

Due to the redundancy of constraints, a superbracket of the 4- $\underline{R} \cup U$ PM can be composed of the four actuation forces $F_{i}(i=1, \ldots, 4)$ in addition to two among the four constraint moments expressed in Eqn. (9). Thus, one can write $\left(\begin{array}{l}4 \\ 2\end{array}\right)=$ $\mathrm{C}_{4}^{2}=6$ superbrackets $S_{j}(j=1, \ldots, 6)$. However, a parallel singularity occurs when the six possible superbrackets vanish simultaneously. For example, the superbracket $S_{1}$ involving the two constraint moments $\underline{\underline{i}} \underline{j}$ and $\underline{\underline{k}} \underline{j}$ takes the form:

$$
S_{1}=[\mathrm{a} \underline{\mathrm{b}}, \mathrm{e} \underline{\mathrm{f}}, \mathrm{c} \underline{\mathrm{d}}, \mathrm{g} \underline{\mathrm{h}}, \underline{\mathrm{i}} \underline{\mathrm{j}}, \underline{\mathrm{k}} \underline{\mathrm{j}}]
$$

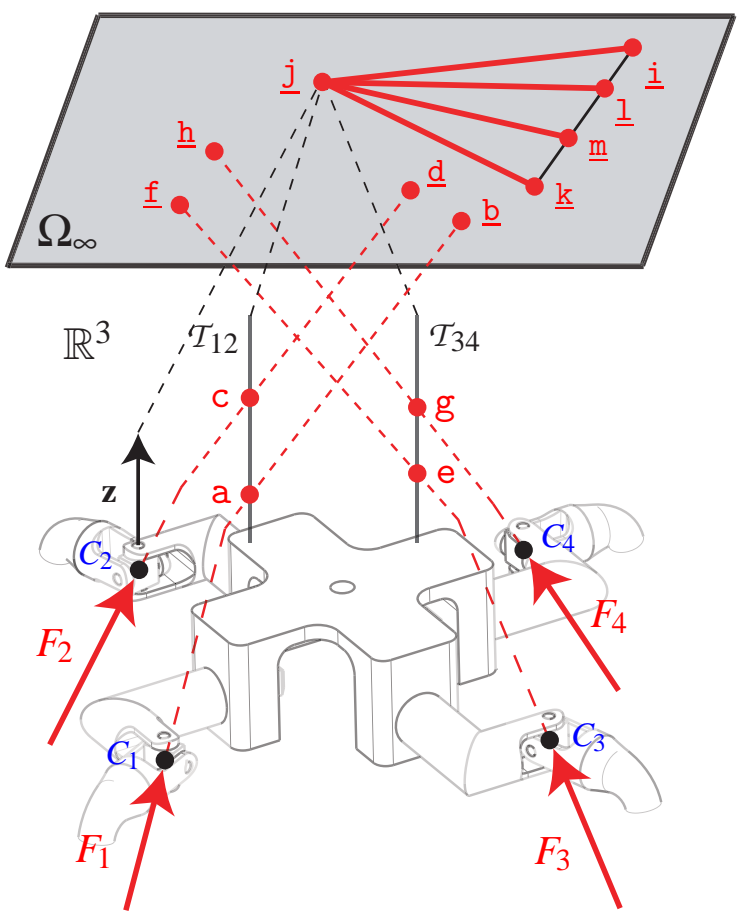

Fig. 5. Wrench graph of the 4-ㅌU PM in $\mathbb{P}_{3}$.

From Eqn. (1), $S_{1}$ can be decomposed into a linear combination of 24 bracket monomials, which leads to only five nonzero monomials as follows:

$$
\begin{aligned}
& S_{1}=-[a \underline{b} \text { e } \underline{f}][c \underline{d} \underline{h} \underline{j}][g \underline{i} \underline{i} \underline{j}]-[a \underline{b} e \underline{d}][\underline{f} g \underline{h} \underline{j}][c \underline{i} \underline{k} \underline{j}] \\
& +[a \underline{b} e \underline{h}][\underline{f} c \underline{d} \underline{j}][g \underline{i} \underline{k} \underline{j}]-[a \underline{b} \underline{f} \underline{h}][\text { e c } \underline{d} \underline{j}][g \underline{i} \underline{k} \underline{j}] \\
& +[a \underline{b} \underline{f} \underline{d}][\operatorname{eg} \underline{h} \underline{j}][c \underline{i} \underline{k} \underline{j}]
\end{aligned}
$$

From Eqn. (2), one has $[g \underline{i} \underline{k} \underline{j}]=[\underline{i} \underline{k} \underline{j}]=[e \underline{i} \underline{k} \underline{j}]$. Furthermore, since points e, $g$ and $j$ belong to the same projective line, namely, $\mathcal{T}_{34}$, the bracket $[\mathrm{eg} \underline{\mathrm{h}} \mathrm{j}]$ is null and as a result: $[\mathrm{a} \underline{\mathrm{b}} \underline{\mathrm{f}} \underline{\mathrm{d}}][\mathrm{e} g \underline{\mathrm{h}} \underline{\mathrm{j}}][\mathrm{c} \underline{\underline{i}} \underline{\mathrm{k}} \underline{j}]=0$. Thus, Eqn. (11) becomes:

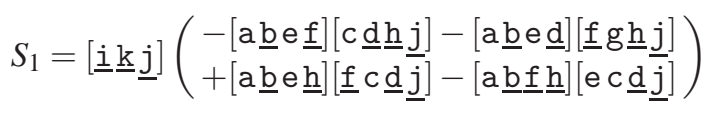

From the syzygies or Grassmann-Plücker relations [13, 27], it follows that:

$$
\begin{aligned}
& {[a \underline{b} e c][\underline{f} \underline{d} \underline{h} \underline{j}]=+[a \underline{b} \text { e } \underline{f}][c \underline{d} \underline{h} \underline{j}]+[a \underline{b} \text { e } \underline{d}][\underline{f} \mathrm{c} \underline{h} \underline{j}]} \\
& +[a \underline{b} e \underline{h}][\underline{f} \underline{d} c \underline{j}]+[a \underline{b} \in \underline{j}][\underline{f} \underline{d} \underline{h} \underline{c}]
\end{aligned}
$$

On the other hand, $[\underline{f} \underline{\mathrm{d}} \underline{\mathrm{h}} \underline{j}]=0$ and $[\underline{\mathrm{f}} \underline{\mathrm{d}} \mathrm{j} \underline{\mathrm{j}}]=-[\underline{\mathrm{f}} \mathrm{c} \underline{\mathrm{d}} \underline{\mathrm{j}}]$. From Eqn. (2) $[\underline{f} g \underline{h} j]=[\underline{f} c \underline{h} j]$. Therefore,

$$
\begin{aligned}
{[a \underline{b} e \underline{j}][\underline{f} \underline{d} \underline{h} c]=} & -[a \underline{b} e \underline{f}][c \underline{d} \underline{h} \underline{j}]-[a \underline{b} e \underline{d}][\underline{f} \mathrm{c} \underline{h} \underline{j}] \\
& +[a \underline{b} \in \hat{h}][\underline{f} c \underline{d}]
\end{aligned}
$$




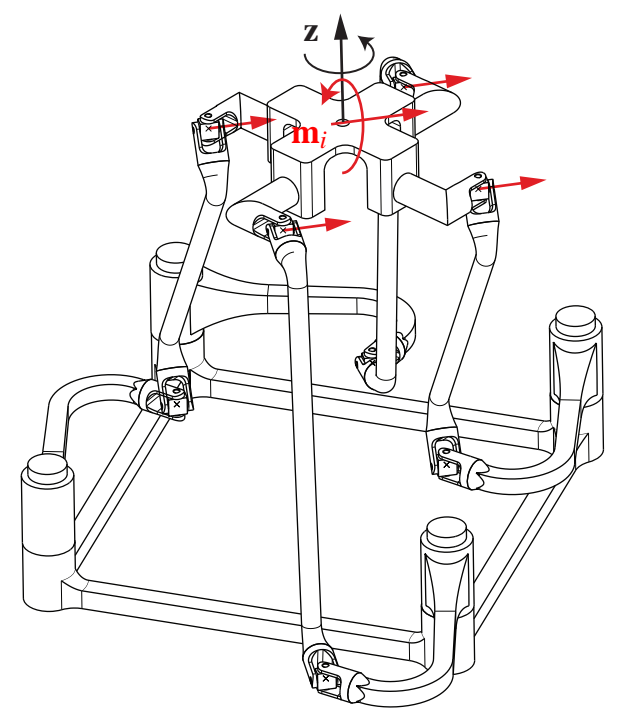

Fig. 6. A constraint singular configuration of the 4-RUU PM.

As a result, Eqn. (12) becomes:

$$
S=\underbrace{[\underline{\underline{i}} \underline{k} \underline{j}]}_{A_{1}} \underbrace{([a \underline{b} e \underline{j}][\underline{f} \underline{\mathrm{d}} \underline{\mathrm{h}} \mathrm{c}]-[\mathrm{a} \underline{\mathrm{b}} \underline{\mathrm{f}} \underline{\mathrm{h}}][\mathrm{e} \mathrm{c} \underline{\mathrm{d}} \underline{\mathrm{j}}])}_{B}
$$

Obviously, in Eqn. (15), term $A_{1}=[\mathrm{g} \underline{\underline{i}} \underline{\mathrm{k}} \underline{\mathrm{j}}]$ depends only on the chosen constraint moments $\underline{\underline{i}} \mathrm{j}$ and $\underline{\mathrm{k}} \mathrm{j}$ whereas term $B$ does not depend on the choice of points $\underline{\underline{i}}$ and $\underline{k}$. Consequently, term $B$ is a common factor of the six possible superbrackets:

$$
S_{j}=A_{j} B \quad ; \quad j=1, \ldots, 6
$$

where $A_{1}=[\underline{\mathrm{i}} \underline{\mathrm{k}} \underline{\mathrm{j}}], A_{2}=[\underline{\mathrm{i}} \underline{\mathrm{l}} \underline{\mathrm{j}}], A_{3}=[\underline{\mathrm{i}} \underline{\mathrm{m}} \underline{\mathrm{j}}], A_{4}=$ $[\underline{\mathrm{k}} \underline{\mathrm{l}} \underline{\mathrm{j}}], A_{5}=[\underline{\mathrm{k}} \underline{\mathrm{m}} \underline{\mathrm{j}}]$ and $A_{6}=[\underline{\mathrm{l}} \underline{\mathrm{m}} \underline{\mathrm{j}}]$.

\subsection{Condition for Constraint Singularities}

Constraint singularities correspond to the degeneracy of the constraint wrench system of the manipulator. In such configurations, the manipulator loses some constraints and, as a result, its moving platform gains one or several DOF. Accordingly, a constraint singularity of the 4- $\underline{\mathrm{RUU}} \mathrm{UM}$ occurs when the four constraint moments $M_{i}(i=1, \ldots, 4)$ form a $n<2$-system, i.e., when all terms $A_{j}(j=1, \ldots, 6)$ expressed in Eqn. (16) vanish simultaneously. Let us consider bracket

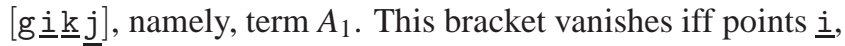
$\underline{\mathrm{k}}$ and $\underline{j}$ belong to the same projective line. Since point $\underline{j}$ corresponds to the fixed direction of vector $\mathbf{z}$, it is a fixed point. Points $\underline{\underline{i}}$ and $\underline{\mathrm{k}}$ correspond to two directions orthogonal to $\mathbf{z}$ and, therefore, these points belong to a line that cannot pass through point $\underline{j}$ unless $\underline{\underline{i}}$ and $\underline{\underline{k}}$ are coincident. Consequently, all terms $A_{j}$ vanish simultaneously iff points $\underline{\underline{i}}, \underline{\mathrm{k}}, \underline{\mathrm{l}}$, and $\underline{\mathrm{m}}$ become all coincident. As a result, the $4-\underline{R} U U$ PM reaches a constraint singularity iff:

$$
\mathbf{m}_{1}\left\|\mathbf{m}_{2}\right\| \mathbf{m}_{3} \| \mathbf{m}_{4}
$$

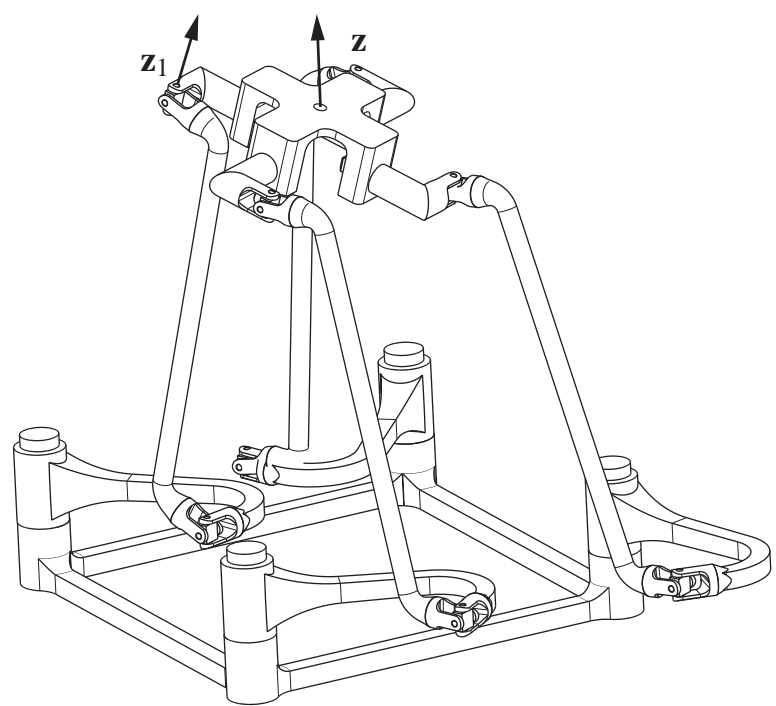

Fig. 7. A coupled motion of the 4-RUU PM.

In such a configuration, the constraint wrench system of the manipulator degenerates into a 1-system and the moving platform gains one extra DOF, namely, the rotation about an axis directed along the common direction of $\mathbf{m}_{i}(i=1, \ldots, 4)$, as shown in Fig. 6. In such a critical configuration, if the moving platform rotates about an axis directed along $\mathbf{z}$, then the robot will come back to a non-singular configuration.

On the other side, in a constraint singular configuration, if the moving platform rotates about an axis directed along $\mathbf{m}_{i}$, the revolute joints attached to the moving platform will no longer be directed along $\mathbf{z}$. As a consequence, the constraint wrench of each limb becomes a wrench of finitepitch (a combination of a force and a moment). In that case, the moving platform has neither pure constraint moments nor pure constraint forces. Moreover, the limbs constrain neither a pure rotation nor a pure translation. Such a configuration is shown in Fig. 7 and corresponds to a coupled motion [29].

\subsection{Conditions for Actuation Singularities}

In this paper, the actuation singularities correspond to configurations in which $\mathbf{J}_{m}$ is rank deficient while the constraint wrench system does not degenerate. In such configurations, the motion of the moving platform becomes uncontrollable, namely, the actuators cannot control the motion of the moving platform. According to Eqn. (16), these singularities are related to the vanishing conditions of term $B$. In order to obtain geometric and vector conditions for actuation singularities, term $B$ is expressed in a more compact form by considering the following bracket simplifications:

1. $[\underline{f} \underline{\mathrm{d}} \underline{\mathrm{h}} \mathrm{c}]=[\mathrm{c} \underline{\mathrm{d}} \underline{\mathrm{f}} \underline{\mathrm{h}}]=[\mathrm{a} \underline{\mathrm{d}} \underline{\mathrm{f}} \underline{\mathrm{h}}]$;

2. Points $a, c$ and $\underline{j}$ belong to the same projective line $\mathcal{T}_{12}$. Thus, $\underline{j}=\mathrm{c}-\mathrm{a}$ and $[\mathrm{a} \underline{\mathrm{b}} \mathrm{e} \underline{\mathrm{j}}]=[(\mathrm{c}-\underline{\mathrm{j}}) \underline{\mathrm{b}} \mathrm{e} \underline{\mathrm{j}}]=[\mathrm{c} \underline{\mathrm{b}} \mathrm{e} \underline{\mathrm{j}}]=[\mathrm{e} \underline{\mathrm{c}} \underline{\mathrm{b}} \underline{\mathrm{j}}]$. 
Table 2. Actuation singularity conditions of the 4- $\underline{R} U U$ PM.

\begin{tabular}{|c|c|c|}
\hline Case & Vector form & Algebraic form \\
\hline (a) & $\mathbf{f}_{3} \| \mathbf{f}_{4}$ & $\underline{\underline{f}} \equiv \underline{\mathrm{h}}$ \\
\hline (b) & $\mathbf{u}=\mathbf{0}$ & $e, c$ and $\underline{j}$ are aligned \\
\hline (c) & $\mathbf{f}_{2} \| \mathbf{f}_{1}$ & $\underline{\mathrm{d}} \equiv \underline{\mathrm{b}}$ \\
\hline (d) & $\left(\mathbf{f}_{3} \times \mathbf{f}_{4}\right) \|(\mathbf{u} \times \mathbf{z})$ & $\underline{f} \underline{h} \equiv \underline{u} \underline{j}$ \\
\hline (e) & $\left(\mathbf{f}_{2} \times \mathbf{f}_{1}\right) \|(\mathbf{u} \times \mathbf{z})$ & $\underline{\mathrm{d}} \underline{\mathrm{b}} \equiv \underline{\mathrm{u}} \underline{j}$ \\
\hline (f) & $\left(\mathbf{f}_{3} \times \mathbf{f}_{4}\right) \|\left(\mathbf{f}_{2} \times \mathbf{f}_{1}\right)$ & $\underline{f} \underline{h} \equiv \underline{\mathrm{d}} \underline{\mathrm{b}}$ \\
\hline (g) & $\left(\mathbf{f}_{3} \times \mathbf{f}_{4}\right)\|(\mathbf{u} \times \mathbf{z})\|\left(\mathbf{f}_{2} \times \mathbf{f}_{1}\right)$ & $\underline{\mathrm{f}} \underline{\mathrm{h}} \equiv \underline{\mathrm{u}} \underline{j} \equiv \underline{\mathrm{d}} \underline{\mathrm{b}}$ \\
\hline (h) & $\left(\left(\mathbf{f}_{3} \times \mathbf{f}_{4}\right) \times(\mathbf{u} \times \mathbf{z})\right) \perp\left(\mathbf{f}_{2} \times \mathbf{f}_{1}\right)$ & $(\underline{\mathrm{f}} \underline{\mathrm{h}} \wedge \underline{\mathrm{u}} \underline{\mathrm{j}}) \in \underline{\mathrm{d}} \underline{\mathrm{b}}$ \\
\hline
\end{tabular}

Accordingly,

$$
\begin{aligned}
& B=[a \underline{d} \underline{f} \underline{h} \underline{]}][\text { c c } \underline{b} \underline{j}]-[a \underline{b} \underline{f} \underline{h}][\text { e c } \underline{d} \underline{j}] \\
& =[\mathrm{a} \underline{\mathrm{d}} \underline{\mathrm{f}} \underline{\mathrm{h}}][\mathrm{e} \text { c } \underline{\dot{b}} \underline{j}] \\
& =(\mathrm{a} \underline{f} \underline{\mathrm{h}}) \wedge(\mathrm{ec} \underline{j}) \wedge(\underline{\mathrm{d}} \underline{\mathrm{b}})
\end{aligned}
$$

where the dotted letters stand for the permuted elements as explained in $[14,27]$. From Eqn. (18), term $B$ is the meet of three geometric entities, namely,

1. $(a \underline{f} \underline{h})$ is a finite plane having $\mathbf{f}_{3} \times \mathbf{f}_{4}$ as normal vector;

2. $(e \subset j)$ is the finite plane containing the finite points e and $\bar{c}$ and the unit vector $\mathbf{z}$. Since plane (ecj) contains lines $\mathcal{T}_{12}$ and $\mathcal{T}_{34}$ (Fig. 5), the line at infinity of plane $($ e c $\underline{j})=\operatorname{span}\left(\mathcal{T}_{12}, \mathcal{T}_{34}\right)$ can be expressed as $(\underline{u} \underline{j})$ where $\underline{\underline{u}}=(\mathbf{u} ; 0)$ and $\mathbf{u}$ is the unit vector of a finite line non-parallel to $\mathbf{z}$ and lying in plane (e c j), i.e., crossing $\mathcal{T}_{12}$ and $\mathcal{T}_{34}$. Accordingly, plane (e c $\left.\underline{j}\right) \overline{\text { has }} \mathbf{u} \times \mathbf{z}$ as normal vector. It should be noted that $\underline{\underline{u}}$ and $\mathbf{u}$ exist unless $\mathcal{T}_{12} \equiv \mathcal{T}_{34}$

3. $(\underline{d} \underline{b})$ is the line at infinity of all parallel finite planes containing the unit vectors $\mathbf{f}_{1}$ and $\mathbf{f}_{2}$, i.e., having $\mathbf{f}_{1} \times \mathbf{f}_{2}$ as normal vector.

An actuation singularity occurs iff term $B$ of Eqn. (18) vanishes. It amounts to the following vector form:

$$
\left(\left(\mathbf{f}_{3} \times \mathbf{f}_{4}\right) \times(\mathbf{u} \times \mathbf{z})\right) \bullet\left(\mathbf{f}_{2} \times \mathbf{f}_{1}\right)=0
$$

From Eqs. (18) and (19), an actuation singularity of the 4$\underline{R} U U$ PM occurs upon each of the following cases:

(a) Plane $(\mathrm{a} \underline{\underline{f}} \underline{\mathrm{h}})$ degenerates, which happens iff $\underline{\underline{f}} \equiv \underline{\mathrm{h}} \Leftrightarrow \mathbf{f}_{3} \|$ $\mathbf{f}_{4}$, i.e., $F_{3}$ and $F_{4}$ are parallel;

(b) Plane (e c j ) degenerates, which happens iff points e, c and $\underline{j}$ are aligned. In that case, $\mathcal{T}_{12} \equiv \mathcal{T}_{34}$ and $\mathbf{u}=\mathbf{0}$;

(c) Line $(\underline{\mathrm{d}} \underline{\mathrm{b}})$ degenerates, which happens iff $\underline{\mathrm{d}} \equiv \underline{\mathrm{b}} \Leftrightarrow \mathbf{f}_{2} \|$ $\mathbf{f}_{1}$

(d) Plane $(\mathrm{a} \underline{f} \underline{h})$ coincides with plane $(\mathrm{e} c j)$. Since point a lies in plane $(\mathrm{ec} \underline{j})$, the condition $(\mathrm{a} \underline{f} \underline{\mathrm{h}}) \equiv(\mathrm{e} c \underline{j})$ amounts to $(\underline{\mathbf{f}} \underline{\mathrm{h}}) \equiv(\underline{\mathrm{u}} \underline{\mathrm{j}})$. In that case, $\left(\mathbf{f}_{3} \times \mathbf{f}_{4}\right) \|(\mathbf{u} \times \mathbf{z})$, i.e., $\mathbf{f}_{3}, \mathbf{f}_{4}, \mathbf{u}$ and $\mathbf{z}$ are orthogonal to a given direction;

(e) Line $(\underline{\mathrm{d}} \underline{\mathrm{b}})$ lies in plane $(\mathrm{a} \underline{\mathbf{f}} \underline{\mathrm{h}})$. In such a case, $\left(\mathbf{f}_{2} \times \mathbf{f}_{1}\right) \|$ $\left(\mathbf{f}_{3} \times \mathbf{f}_{4}\right)$

(f) Line ( $\underline{\mathrm{d}} \underline{\mathrm{b}})$ lies in plane $(\mathrm{e} \mathrm{c} \underline{\mathrm{j}})$. In such a case, $\left(\mathbf{f}_{2} \times \mathbf{f}_{1}\right) \|$ $(\mathbf{u} \times \mathbf{z})$;

(g) The intersection line of planes $(\mathrm{a} \underline{\mathrm{f}} \underline{\mathrm{h}})$ and $(\mathrm{e} \subset \underline{j})$ coincides with line $(\underline{d} \underline{b})$. Since planes $(a \underline{f} \underline{h}$ ) and (ec j) contain point $a$, they intersect at a line at infinity iff they coincide. Accordingly, condition (g) amounts to $(\underline{\mathbf{f}} \underline{\mathrm{h}}) \equiv(\underline{\mathrm{u}} \underline{\mathrm{j}}) \equiv(\underline{\mathrm{d}} \underline{\mathrm{b}})$, i.e., $\left(\mathbf{f}_{3} \times \mathbf{f}_{4}\right)\|(\mathbf{u} \times \mathbf{z})\|\left(\mathbf{f}_{2} \times \mathbf{f}_{1}\right) ;$

(h) Let us consider the general case of Eqn. (19), namely, the intersection line of planes $(\mathrm{a} \underline{\underline{f}} \underline{\mathrm{h}})$ and $(\mathrm{e} c \underline{j})$ crosses line $(\underline{d} \underline{b})$. If planes $(a \underline{f} \underline{h})$ and $(e c \underline{j})$ are not coincident (condition $(\mathbf{d}))$, then they will intersect at a finite line $\mathcal{D}$ directed along $\mathbf{n}=\left(\mathbf{f}_{3} \times \mathbf{f}_{4}\right) \times(\mathbf{u} \times \mathbf{z})$. Thus, the point at infinity $\underline{\mathrm{n}}=(\mathbf{n} ; 0)$ of line $\mathcal{D}$ is the intersection point of lines $(\underline{f} \underline{h})$ and $(\underline{u} j)$. The finite line $\mathcal{D}$ crosses line $(\underline{\mathrm{d}} \underline{\mathrm{b}})$ iff $\underline{n} \in(\underline{\mathrm{d}} \underline{\mathrm{b}})$. In that case, the lines at infinity ( $\underline{\mathrm{f}} \underline{\mathrm{h}}),(\underline{\mathrm{d}} \underline{\mathrm{b}})$ and ( $\underline{u} \underline{j})$ intersect at point $\underline{n}$. As a result, $\mathbf{n}$ is orthogonal to $\left(\mathbf{f}_{2} \times \mathbf{f}_{1}\right),\left(\mathbf{f}_{3} \times \mathbf{f}_{4}\right)$ and $(\mathbf{u} \times \mathbf{z})$.

On the other hand, as mentioned previously, $\mathbf{u}$ is the unit vector of any finite line non-parallel to $\mathbf{z}$ and crossing $\mathcal{T}_{12}$ and $\mathcal{T}_{34}$. Thus, the condition $\mathbf{n} \perp(\mathbf{u} \times \mathbf{z})$ is true for all possible vectors $\mathbf{u}$ iff $\underline{n} \equiv j$, namely, if $\mathbf{n} \| \mathbf{z}$. As a result, point $\underline{j}$ belongs to lines $(\underline{\mathrm{d}} \underline{\mathrm{b}})$ and $(\underline{\mathrm{f}} \underline{\mathrm{h}})$. We know that in a general configuration, two forces $F_{1}$ and $F_{2}$ are two skew lines. However, if $\underline{j} \in(\underline{\mathrm{d}} \underline{\mathrm{b}})$, then any plane defined by two finite lines directed along $\mathbf{f}_{1}$ and $\mathbf{f}_{2}$ will contain the unit vector $\mathbf{z}$. Moreover, there is a finite line $\mathcal{T}_{12}$ directed along $\mathbf{z}$ that crosses $F_{1}$ and $F_{2}$. Consequently, $F_{1}$ and $F_{2}$ are coplanar. Likewise, $F_{3}$ and $F_{4}$ are coplanar.

Finally, condition (h) implies that $F_{1}$ and $F_{2}$ lie in a plane containing $\mathcal{T}_{12}$ and, in turn, $F_{3}$ and $F_{4}$ lie in a plane containing $\mathcal{T}_{34}$.

All possible cases of Eqn. (19) are expanded in Table 2. It should be noted that Eqn. (19) is obtained by considering two lines $\mathcal{T}_{12}$ (crossing $F_{1}$ and $F_{2}$ ) and $\mathcal{T}_{34}$ (crossing $F_{3}$ and $F_{4}$ ). Vector $\mathbf{u}$ in Eqn. (19) could be written as $\mathbf{u}_{12}^{34}$, i.e., the unit vector of a line non-parallel to $\mathbf{z}$ and crossing lines $\mathcal{T}_{12}$ and $\mathcal{T}_{34}$. Accordingly, since a line $\mathcal{T}_{i j}$ exists between each pair of forces $F_{i}$ and $F_{j}$, the vector form of actuation singularities can be generalized as follows:

$$
\left(\left(\mathbf{f}_{i} \times \mathbf{f}_{j}\right) \times\left(\mathbf{f}_{k} \times \mathbf{f}_{l}\right)\right) \bullet\left(\mathbf{u}_{i j}^{k l} \times \mathbf{z}\right)=0
$$

where $(i, j, k, l), i \neq j \neq k \neq l$, is a permutation of $(1,2,3,4)$ and $\mathbf{u}_{i j}^{k l}$ is the unit vector of a line non-parallel to $\mathbf{z}$ and crossing $\mathcal{T}_{i j}$ and $\mathcal{T}_{k l}$. Table 3 enumerates the general singularity conditions obtained with GCA for the 4-RUU PM as well as their correspondence with GG, which is developed in the next section. 
Table 3. Correspondence between GCA and GG.

\begin{tabular}{l|l|l}
\hline \hline Case & \multicolumn{1}{|c|}{$\begin{array}{c}\text { Singularity condition } \\
\text { GCA }(i \neq j \neq k \neq l)\end{array}$} & $\begin{array}{c}\text { Corresponding case } \\
\text { of GG (Table 1) }\end{array}$ \\
\hline (a) & $\mathbf{f}_{i} \| \mathbf{f}_{j}$ & condition (3b) \\
\hline (b) & $\mathbf{u}_{i j}^{k l}=\mathbf{0}$ & condition (5b) \\
\hline (c) & $\left(\mathbf{f}_{i} \times \mathbf{f}_{j}\right) \|\left(\mathbf{u}_{i j}^{k l} \times \mathbf{z}\right)$ & condition (5b) \\
\hline (d) & $\left(\mathbf{f}_{i} \times \mathbf{f}_{j}\right) \|\left(\mathbf{f}_{k} \times \mathbf{f}_{l}\right)$ & conditions (5b) and (3d) \\
\hline$(\mathbf{e})$ & $\left(\mathbf{f}_{i} \times \mathbf{f}_{j}\right)\left\|\left(\mathbf{u}_{i j}^{k l} \times \mathbf{z}\right)\right\|\left(\mathbf{f}_{k} \times \mathbf{f}_{l}\right)$ & condition (5b) \\
\hline (f) & $\left(\left(\mathbf{f}_{i} \times \mathbf{f}_{j}\right) \times\left(\mathbf{f}_{k} \times \mathbf{f}_{l}\right)\right) \perp\left(\mathbf{u}_{i j}^{k l} \times \mathbf{z}\right)$ & condition (5a) \\
\hline (g) & $\mathbf{m}_{i}\left\|\mathbf{m}_{j}\right\| \mathbf{m}_{k} \| \mathbf{m}_{l}$ & conditions 1 and (5b) \\
\hline \hline
\end{tabular}

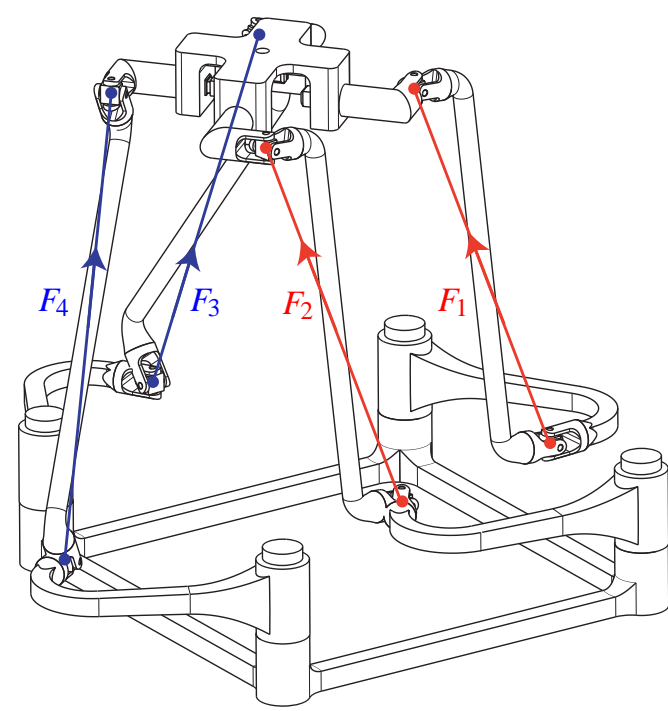

7 Correspondence between Grassmann-Cayley Algebra and Grassmann Geometry

There is a complementary aspect between GCA and GG for the singularity analysis of PMs. Mostly, some conditions obtained with one of these approaches may not be easy to grasp with the other one and vice versa. Therefore, in this section, a wrench graph for each singularity condition obtained with GCA is represented. Based on this wrench graph, the GG is used to identify the rank deficiency of $\mathbf{J}_{m}$. Let us consider the following screw subspaces:

1. $\mathcal{K}=\operatorname{span}\left(F_{1}, F_{2}, F_{3}, F_{4}, M_{1}, M_{2}, M_{3}, M_{4}\right)$;

2. $\mathcal{K}_{12}=\operatorname{span}\left(F_{1}, F_{2}, M_{1}, M_{2}, M_{3}, M_{4}\right)$;

3. $\mathcal{F}=\operatorname{span}\left(F_{1}, F_{2}, F_{3}, F_{4}\right)$;

4. $\mathcal{M}=\operatorname{span}\left(M_{1}, M_{2}, M_{3}, M_{4}\right)$.

From (2b1), since the four lines at infinity $M_{1}, M_{2}, M_{3}$ and $M_{4}$ pass through point $\mathrm{j}$, they generate in a general configuration a planar pencil of lines at infinity. In turn, in a general configuration the four actuation forces are mutually skew and span a linear line variety of dimension 4 . Thus, in a nonsingular posture $\operatorname{dim}(\mathscr{M})=2, \operatorname{dim}(\mathcal{F})=4, \operatorname{dim}\left(\mathcal{K}_{12}\right)=4$ and $\operatorname{dim}(\mathcal{K})=6$. However, a parallel singularity occurs whenever $\mathcal{K}$ becomes of dimension lower than 6 . Here, the degeneracy of $\mathcal{K}$ for the singularity conditions revealed by GCA in Table 3, is examined by using Table 1 and the subclasses (2b1), (2b2) and (3d1).

(a) $\left(\mathbf{f}_{i} \| \mathbf{f}_{j}\right)$. Without loss of generality, let us consider that $\mathbf{f}_{1} \| \mathbf{f}_{2}$, i.e., $\underline{\mathrm{b}} \equiv \underline{\mathrm{d}}$, as depicted in Fig. 10(a). In that case, from (2b2), one has: $\operatorname{span}\left(F_{1}, F_{2}\right)=\operatorname{span}\left(F_{1}, L\right)$ where $\mathcal{L}=\underline{\mathrm{b}} \boldsymbol{j}$. Moreover, the line at infinity $\mathcal{L}$ passes through point $j$ and lies in the planar pencil containing the four constraint moments. Accordingly, the screw subspace $\mathcal{K}_{12}$ - which is generally of dimension 4 - becomes of dimension 3 . In other words,

1. From (2b1), $\mathcal{L}$ lies in the planar pencil at infinity through point $\underline{j}$ generated by the four constraint moments;

2. From (2b2), $L$, being the line at infinity of plane $(\mathrm{a} \underline{\mathrm{b}} \mathrm{c})$, lies in the planar pencil $\operatorname{span}\left(F_{1}, F_{2}\right)$ of lines in plane $(\mathrm{a} \underline{\mathrm{b}} \mathrm{c})$ passing through point $\underline{\mathrm{b}}$.

Fig. 8. An actuation singularity: the two actuation forces $F_{1}$ and $F_{2}$ are parallel.

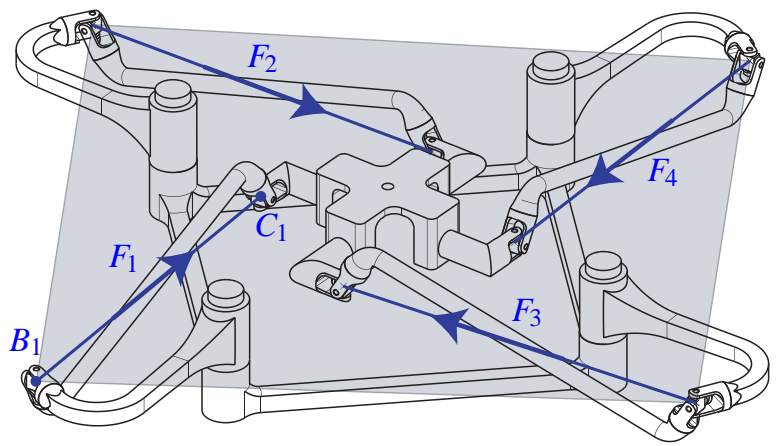

Fig. 9. An actuation singularity: all actuation forces are coplanar, condition (3d) of GG.

Consequently, such a configuration $\left(\mathbf{f}_{1} \| \mathbf{f}_{2}\right)$, exemplified in Fig. 8, corresponds to condition (3b) of GG.

(b) $\left(\mathbf{u}_{i j}^{k l} \times \mathbf{z}\right)=\mathbf{0}$. In such a case, one has: $\mathcal{T}_{i j} \equiv \mathcal{T}_{k l}$ and $\left(P_{i} \cap P_{j}\right) \equiv\left(\mathcal{P}_{k} \cap \mathscr{P}_{l}\right)$ as illustrated in Fig. 10(b). As a result, the four planes $P_{i}(i=1, \ldots, 4)$ of normal vectors $\left(\mathbf{z} \times \mathbf{f}_{i}\right)=\mathbf{m}_{i}$ intersect at a common projective line $\mathcal{T}_{i j} \equiv$ $\mathcal{T}_{k l}$ passing through point $j$ and coplanar with each of the four actuation forces. Consequently, the latter line crosses the four constraint moments (the planar pencil at infinity through point $j$ ) as well as the four actuation forces. Thus, this singularity corresponds to a singular complex, i.e., condition (5b) of GG.

(c) $\left(\mathbf{f}_{i} \times \mathbf{f}_{j}\right) \|\left(\mathbf{u}_{i j}^{k l} \times \mathbf{z}\right)$. Without loss of generality, let us consider an example of this case when $\left(\mathbf{f}_{1} \times \mathbf{f}_{2}\right) \|(\mathbf{u} \times \mathbf{z})$ where $\mathbf{u}=\mathbf{u}_{12}^{34}$. In such a case, $(\underline{b} \underline{d}) \equiv(\underline{\mathbf{u}} \underline{j})$. Thus, plane $(e \subset j)=\operatorname{span}\left(\mathcal{T}_{12}, \mathcal{T}_{34}\right)$, whose line at infinity is $(\underline{u} j)$, includes points $\underline{b}$ and $\underline{d}$ in addition to points $a, c, e$ and $g$. Consequently, the two actuation forces $F_{1}$ and $F_{2}$ lie in plane (e c j ) and cross line $\mathcal{T}_{34}$ directed along $\mathbf{z}$. Therefore, line $\overline{\mathcal{T}}_{34}$ intersects the four actuation forces and the four constraint moments as shown in Fig. 10(c). As a result, such a configuration corresponds to a singu- 


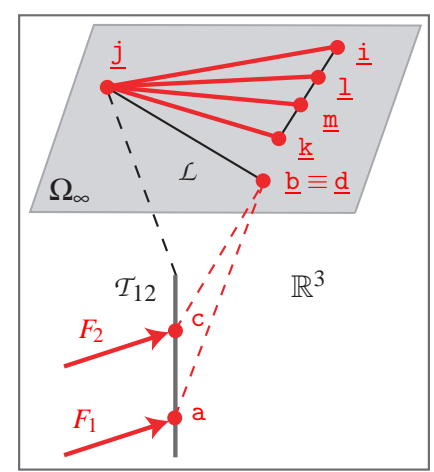

(a)

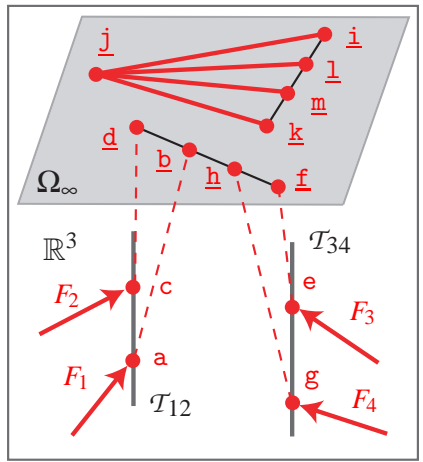

(d)

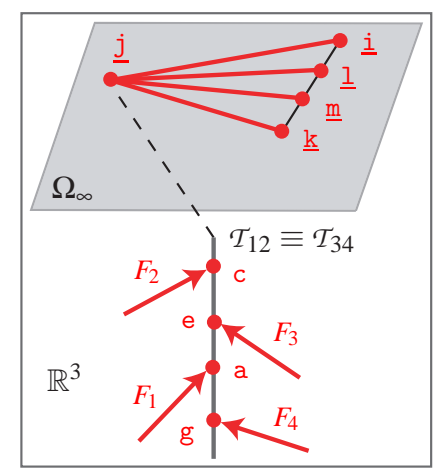

(b)

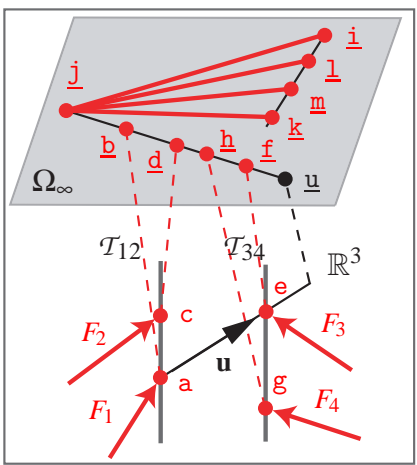

(e)

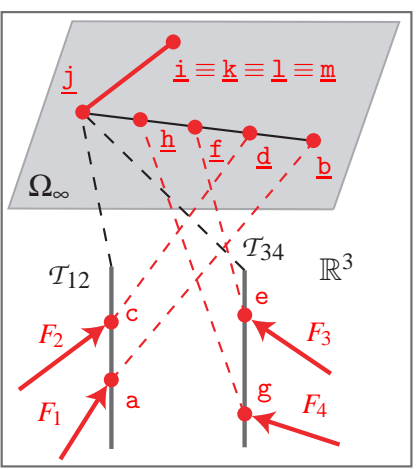

(g)

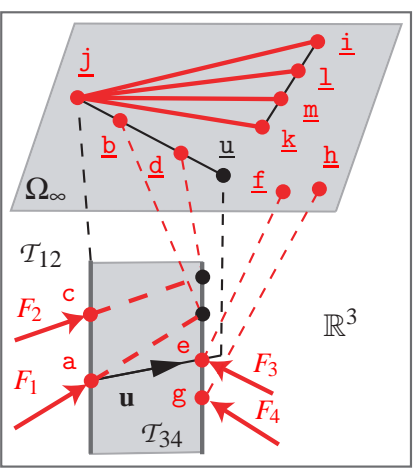

(c)

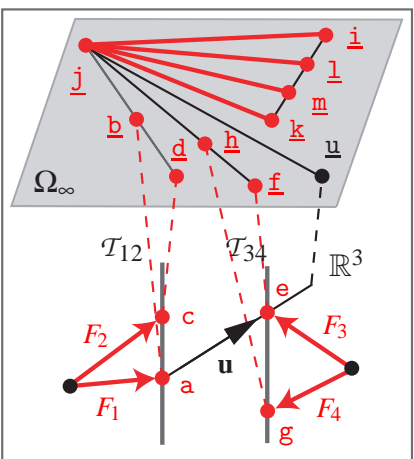

(f)

Fig. 10. Wrench graph of the 4-RUU PM for the singularity conditions given in table 3 .

lar complex, i.e., condition (5b) of GG.

(d) $\left(\mathbf{f}_{i} \times \mathbf{f}_{j}\right) \|\left(\mathbf{f}_{k} \times \mathbf{f}_{l}\right)$. Here, two cases are possible:

1. The four actuation forces are not coplanar but are orthogonal to a given direction. In such a case, $\underline{\mathrm{b}}, \underline{\mathrm{d}}, \underline{\mathrm{f}}$ and $\underline{\mathrm{h}}$ belong to a given line at infinity, namely, $(\underline{\mathrm{b}} \underline{\mathrm{d}}) \equiv(\underline{\mathrm{f}} \underline{\mathrm{h}})$ as depicted in Fig. 10(d). Obviously, the latter line crosses the four actuation forces. Furthermore, since it is a line at infinity, it also crosses the four constraint moments. Consequently, such a configuration corresponds to a singular complex, i.e., condition (5b) of GG;

2. The four actuation forces are coplanar. In that case, the screw subspace $\mathcal{F}$ becomes of dimension 3 , which corresponds to condition (3d) of GG, all lines in a plane. Such a configuration is illustrated in Fig. 9. (e) $\left(\mathbf{f}_{i} \times \mathbf{f}_{j}\right)\left\|\left(\mathbf{u}_{i j}^{k l} \times \mathbf{z}\right)\right\|\left(\mathbf{f}_{k} \times \mathbf{f}_{l}\right)$. As mentioned previously, this condition amounts to $(\underline{f} \underline{\mathrm{h}}) \equiv(\underline{\mathrm{u}} \underline{j}) \equiv(\underline{\mathrm{d}} \underline{\mathrm{b}})$. In that case, points $\underline{f}, \underline{h}, \underline{u}, \underline{j}, \underline{d}$ and $\underline{b}$ are aligned, as shown in Fig. 10(e). Therefore, such a configuration corresponds to a singular complex, i.e., condition (5b) of GG.

(f) $\left(\left(\mathbf{f}_{i} \times \mathbf{f}_{j}\right) \times\left(\mathbf{f}_{k} \times \mathbf{f}_{l}\right)\right) \perp\left(\mathbf{u}_{i j}^{k l} \times \mathbf{z}\right)$. This is the general case of Eqn. (20). Without loss of generality, let us consider that $\left(\left(\mathbf{f}_{1} \times \mathbf{f}_{2}\right) \times\left(\mathbf{f}_{3} \times \mathbf{f}_{4}\right)\right) \perp\left(\mathbf{u}_{12}^{34} \times \mathbf{z}\right)$. As mentioned previously, in that case, $F_{1}$ and $F_{2}$ lie in a plane containing $\mathcal{T}_{12}$ and, in turn, $F_{3}$ and $F_{4}$ lie in a plane containing $\mathcal{T}_{34}$, as shown in Fig. 10(f). As a result, the lines at infinity of planes $\operatorname{span}\left(F_{1}, F_{2}\right)$ and $\operatorname{span}\left(F_{3}, F_{4}\right)$ as well as the lines at infinity denoting the constraint moments intersect all at point $j$. Thus, the foregoing lines at infinity form a planar pencil. Following [16,21], in such a configuration, the eight wrenches of the 4-RUU PM lie in a 
non-singular complex, i.e., condition (5a) of GG.

(g) $\left(\mathbf{m}_{i}\left\|\mathbf{m}_{j}\right\| \mathbf{m}_{k} \| \mathbf{m}_{l}\right)$. This is the condition for constraint singularities. In that case, two interpretations are possible:

1. The screw subspace $\mathcal{M}$, which is generally a planar pencil, degenerates into a single line, i.e., condition 1 of GG (Fig. 10(g));

2. Planes $\mathcal{P}_{i}(i=1, \ldots, 4)$ have a common normal vector $\mathbf{m}_{i}=\mathbf{z} \times \mathbf{f}_{i}$. Those planes are thus all parallel and have a common line at infinity containing points $\underline{j}, \underline{b}, \underline{d}, \underline{f}$ and $\underline{h}$. This line crosses all actuation forces and all constraint moments. Hence, such a configuration corresponds to a singular complex, i.e., condition (5b) of GG.

Consequently, the constraint singularities of the 4$\underline{\mathrm{R} U U} \mathrm{PM}$ correspond to conditions 1 and (5b) of GG.

\section{Singularity conditions of 3T1R PMs with ILS}

The singularity analysis of the 4-RUU PM performed in Sec. 6 leads to two singularity conditions given by Eqs. (17) and (20). These conditions were obtained from the wrench graph of the PM obtained in Fig. 5 and based on the existence of a finite line parallel to $\mathbf{z}$ crossing two actuation forces of two limbs of the PM. Since this is true in a general configuration for all architectures of Fig. 1, a similar wrench graph can be obtained for each architecture. As a consequence, the two singularity conditions are true for the eleven architectures shown in Fig. 1.

Moreover, the actuation singularity condition obtained in Eqn. (20) is valid for 3T1R PMs whose actuation forces are four mutually skew lines. Indeed, a finite line $\mathcal{T}_{i j}$ directed along $\mathbf{z}$ that crosses two actuation forces can be found unless one of the forces is along $\mathbf{z}$ or the two forces are parallel and lie in a plane that does not contain the $\mathbf{z}$ direction. Accordingly, Eqn. (20) is the actuation singularity condition for the general case of 3T1R PMs with ILS which is the case for the eleven architectures of Fig. 1 obtained from the type synthesis of four-legged 3T1R PMs with ILS performed in [6].

The eleven architectures of Fig. 1 are compared below in terms of both constraint and actuation singularities.

\subsection{Constraint Singularities}

From Eqn. (17) all architectures of Fig. 1 exhibit a constraint singularity whenever the axes of the revolute joints non-parallel to $\mathbf{z}$ become parallel to each other, namely, $\mathbf{m}_{1}\left\|\mathbf{m}_{2}\right\| \mathbf{m}_{3} \| \mathbf{m}_{4}$. However, it can be noticed that when the last joints of the legs of the PM are not directed along $\mathbf{z}$ the four vectors $\mathbf{m}_{i}$ are fixed with respect to each other, namely, none can be parallel to another one in any robot configuration.

As a result, the PMs presented in Figs. 1(b), 1(d), 1(f) and 1(j) are free of constraint singularities.

\subsection{Actuation Singularities}

The condition for actuation singularities obtained in Eqn. (20) involves the unit vector of the four actuation forces plus the normal vector of the plane defined by two transversal lines along $\mathbf{z}$ between two actuation forces. However, the eleven architectures of Fig. 1 are classified into two types as proved in Sec. 3.2:

Type 1: Figs. 1(a)-1(d) The actuation force of the $i$ th leg is directed along the intersection line of planes $\mathcal{P}_{i}$ and $\mathcal{V}_{i}$. In that case, the actuation singularity condition is given in Eqn. (20).

Type 2: Figs. 1(e)-1(k) The actuation force of the $i$ th leg is parallel to $\mathbf{m}_{i}$. In that case, the actuation singularity condition can be written as:

$$
\left(\left(\mathbf{m}_{i} \times \mathbf{m}_{j}\right) \times\left(\mathbf{m}_{k} \times \mathbf{m}_{l}\right)\right) \bullet\left(\mathbf{u}_{i j}^{k l} \times \mathbf{z}\right)=0
$$

where $(i, j, k, l), \quad i \neq j \neq k \neq l$, is a permutation of $(1,2,3,4)$.

It can be noticed that for the architectures of Figs. 1(b) and 1(d) none of the four actuation forces (directed along $\mathbf{m}_{i}$ ) can be parallel to another one in any robot configuration.

\section{Conclusions}

In this paper, the singularity conditions of 3T1R Parallel Manipulators (PMs) with identical limb structures (ILS) were investigated through the singularity analysis of the 4$\underline{R} U$ U PM. This singularity analysis was performed using Grassmann-Cayley Algebra (GCA) and Grassmann Geometry (GG).

First, the constraint analysis was performed using screw theory. Then a wrench graph that represents the wrenches of the 4-RUU PM in the 3-dimensional projective space was obtained. Accordingly, a superbracket was formulated and analyzed in order to provide geometric conditions for singular configurations as well as a compact vector expression for the singularity locus, which is difficult to assess using classical linear algebra tools. Then, a wrench graph representation for each singularity condition was obtained to emphasize the linear dependence of the constraint and actuation wrenches. This paper showed that the concept of wrench graph is very useful to highlight the correspondence between GCA and GG for the singularity analysis of lower-mobility PMs.

Finally, the singularity conditions of the 4-RUU PM were applied to eleven architectures of 3T1R PMs with ILS to show the general aspect of the results. The foregoing architectures were compared in terms of constraint and actuation singularities.

\section{Achnowledgements}

The authors would like to acknowledge the French "Agence Nationale de la Recherche" (Project "SiRoPa", SIngularités des RObots PArallèles), the financial support of 
the Natural Sciences and Engineering Research Council of Canada (NSERC) and the Canada Research Chair program.

Finally, the authors would like to thank Pierre-Luc Richard and Thierry Laliberte for the CAD models of the 4-ㅡU parallel manipulator.

\section{References}

[1] Zlatanov, D., Bonev, I., and Gosselin, C. M., 2002. "Constraint Singularities of Parallel Mechanisms". In IEEE International Conference on Robotics and $\mathrm{Au}-$ tomation, pp. 496-502.

[2] Zlatanov, D., Fenton, R. G., and Benhabib, B., 1994. "Singularity Analysis of Mechanisms and Robots Via a Velocity-Equation Model of the Instantaneous Kinematics". In IEEE International Conference on Robotics and Automation, pp. 986-991.

[3] Ball., R. S., 1900. A Treatise On the Theory of Screws. Cambridge University Press, Cambridge, CA.

[4] Waldron, K. J., 1969. "The Mobility of Linkages". PhD Thesis, Stanford University, Cambridge, CA.

[5] Hunt, K. H., 1978. Kinematic Geometry of Mechanisms. Clarendon Press, Oxford.

[6] Kong, X., and Gosselin, C., 2007. Type Synthesis of Parallel Mechanisms, Vol. 33. Springer, Heidelberg.

[7] Amine, S., Kanaan, D., Caro, S., and Wenger, P., 2010. "Constraint and Singularity Analysis of LowerMobility Parallel Manipulators with Parallelogram Joints". In ASME 2010 International Design Engineering Technical Conferences, no. 28483 in DETC2010.

[8] Joshi, S. A., and Tsai, L. W., 2002. "Jacobian Analysis of Limited-DOF Parallel Manipulators". ASME Journal of Mechanical Design, 124(2), June, pp. 254-258.

[9] Gosselin, C., and Angeles, J., 1990. "Singularity Analysis of Closed-Loop Kinematic Chains". IEEE Transactions on Robotics and Automation, 6(3), pp. 281290.

[10] Fang, Y., and Tsai, L. W., 2002. "Structure Synthesis of a Class of 4-DoF and 5-DoF Parallel Manipulators with Identical Limb Structures". The International Journal of Robotics Research, 21(9), pp. 799-810.

[11] Conconi, M., and Carricato, M., 2008. “A New Assessment of Singularities of Parallel Kinematic Chains”. In Advances in Robot Kinematics: Analysis and Design, J. Lenarcic and P. Wenger, eds., Springer, pp. 3-12.

[12] Downing, D., Samuel, A., and Hunt, K., 2002. "Identification of the Special Configurations of the Octahedral Manipulator using the Pure Condition”. The International Journal of Robotics Research, 21(2), pp. 147159.

[13] Ben-Horin, P., and Shoham, M., 2006. "Singularity Analysis of a Class of Parallel Robots Based on Grassmann-Cayley Algebra". Mechanism and Machine Theory, 41(8), pp. 958-970.

[14] Ben-Horin, P., and Shoham, M., 2009. "Application of Grassmann-Cayley Algebra to Geometrical Interpretation of Parallel Robot Singularities". The International Journal of Robotics Research, 28(1), pp. 127-141.
[15] Kanaan, D., Wenger, P., Caro, S., and Chablat, D., 2009. "Singularity Analysis of Lower-Mobility Parallel Manipulators using Grassmann-Cayley Algebra". IEEE Transactions on Robotics, 25, pp. 995-1004.

[16] Merlet, J. P., 1988. Parallel Manipulators. Part 2: Theory. Singular Configurations and Grassmann Geometry. Technical Report 791, INRIA, Sophia Antipolis, France.

[17] Merlet, J. P., 1989. "Singular Configurations of Parallel Manipulators and Grassmann Geometry". The International Journal of Robotics Research, 8(5), pp. 45-56.

[18] Merlet, J. P., 2006. Parallel Robots, Vol. 128 of Solid Mechanics and Its Applications. Springer.

[19] Mbarek, T., Lonij, G., and Corves, B., 2007. "Singularity Analysis of a Fully Parallel Manipulator with FiveDegrees-of-Freedom Based on Grassmann Line Geometry". In 12th IFToMM World Congress.

[20] Masouleh, M. T., and Gosselin, C., 2011. "Singularity Analysis of 5-RPUR Parallel Mechanisms (3T2R)". The International Journal of Advanced Manufacturing Technology, 57(9-12), pp. 1107-1121.

[21] St-Onge, B. M., and Gosselin, C. M., 2000. "Singularity Analysis and Representation of the General Gough-Stewart Platform". The International Journal of Robotics Research, 19(3), March, pp. 271-288.

[22] Amine, S., Kanaan, D., Caro, S., and Wenger, P., 2010. "Singularity Analysis of Lower-Mobility Parallel Robots with an Articulated Nacelle". In Advances in Robot Kinematics: Motion in Man and Machine 2010, Part 5, Springer, pp. 273-282.

[23] Amine, S., Masouleh, M. T., Caro, S., Wenger, P., and Gosselin, C., 2011. "Singularity Analysis of 5-DOF Parallel Mechanisms 3T2R using Grassmann-Cayley Algebra". In 13th IFToMM World Congress in Mechanism and Machine Science.

[24] Caro, S., Khan, W. A., Pasini, D., and Angeles, J., 2010. "The Rule-based Conceptual Design of the Architecture of Serial Schönflies-motion Generators". Mechanism and Machine Theory, 45(2), pp. 251-260.

[25] Kong, X., and Gosselin, C., 2004. "Type Synthesis of 3T1R 4-DOF Parallel Manipulators Based on Screw Theory". IEEE Transactions on Robotics and Automation, 20(2), pp. 181-190.

[26] Gogu, G., 2007. "Structural Synthesis of FullyIsotropic Parallel Robots with Schoenflies Motions via Theory of Linear Transformations and Evolutionary Morphology". European Journal of MechanicsA/Solids, 26(2), pp. 242-269.

[27] White, N. L., 2005. Handbook of Geometric Computing, Vol. VIII. Springer, Berlin Heidelberg, ch. Grassmann-Cayley Algebra and Robotics Applications, pp. 629-656.

[28] McMillan, T., 1990. "Invariants of Antisymmetric Tensors". PhD Thesis, University of Florida, Gainesville, Florida, USA.

[29] Zlatanov, D., Bonev, I., and Gosselin, C. M., 2002. "Constraint Singularities as C-Space Singularities". In 8th International Symposium on Advances in Robot 
Kinematics, pp. 183-192. 